\title{
Modeling Long-Period Swell in Southern California: Practical Boundary Conditions from Buoy Observations and Global Wave Model Predictions ${ }^{\mathscr{O}}$
}

\author{
Sean C. Crosby, William C. O’Reilly, and Robert T. GuZA \\ Scripps Institution of Oceanography, La Jolla, California
}

(Manuscript received 1 February 2016, in final form 11 April 2016)

\begin{abstract}
Accurate, unbiased, high-resolution (in space and time) nearshore wave predictions are needed to drive models of beach erosion; coastal flooding; and alongshore transport of sediment, biota, and pollutants. On sheltered shorelines, wave predictions are sensitive to the directions of onshore propagating waves, and nearshore model prediction error is often dominated by directional uncertainty offshore. Here, regional wave model skill in highly sheltered Southern California is compared for different offshore boundary conditions created from offshore buoy observations and global wave model hindcasts [NOAA WaveWatch III (WW3)]. Spectral ray-tracing methods are used to transform incident offshore swell $(0.04-0.09 \mathrm{~Hz})$ energy at high directional resolution $\left(1^{\circ}\right)$. Model skill is assessed for predictions (wave height, direction, directional spread, and alongshore radiation stress) at 16 nearshore buoy sites between 2000 and 2009. Buoy-derived boundary conditions using various estimators (maximum entropy, maximum smoothness) have similar skill and all outperform WW3-derived boundary conditions. A new method for estimating offshore boundary conditions, CMB-ADJ, combines buoy observations with WW3 predictions. Although CMB-ADJ skill is comparable to buoy-only methods, it may be more robust in varying regions and wave climatologies, and will benefit from future improvements in global wave model (GWM) predictions. A case study at Oceanside Harbor shows strong sensitivity of alongshore sediment transport estimates to the boundary condition method. However, patterns in alongshore gradients of transport (e.g., the location of model accretion and erosion zones) are similar across methods. Weak, tidally modulated coastal reflection is evident in both shallow and deep buoy observations, and significantly increases the observed directional spread.
\end{abstract}

\section{Introduction}

Nearshore waves impact port and waterway operations, beach recreation, boating safety, and contribute to coastal inundation. Over decades, nearshore wave energy shapes the coastline by eroding beaches and transporting sediment alongcoast. Accurate high-resolution wave predictions are needed in hindcast, real-time, and forecast modes. Operational global wave models (GWMs) have good skill due to recent improvements of model parameterizations (Ardhuin et al. 2010), development of obstruction grids (Chawla and Tolman 2008), faster

Supplemental information related to this paper is available at the Journals Online website: http://dx.doi.org/10.1175/ JTECH-D-16-0038.s1.

Corresponding author address: Sean C. Crosby, Scripps Institution of Oceanography, 8622 Kennel Way, La Jolla, CA 92037. E-mail: sccrosby@ucsd.edu computers, and increasing satellite global wind coverage. However, GWM spatial resolution is typically too coarse to properly resolve local islands, shoals, and rough coastline features, for example, Southern California (SC) shown in Fig. 1.

Higher-resolution nearshore wave predictions are obtained by dynamical downscaling, that is, the use of regional nonlinear third-generation wave models, for example, García-Medina et al. (2013). Though large regions and long time series can require significant computational resources, statistical and/or hybrid downscaling methods can reduce computational expense (Camus et al. 2011, 2013). Hybrid methods model a small set of representative wave conditions, forming a lookup table that is used to create continuous predictions. When the local wave climatology is relatively simple, for example, limited exposure to large ocean basis, hybrid methods work well. However, in regions with wide exposure and many energy sources, the model lookup table required to adequately represent possible conditions may become unmanageably large. 


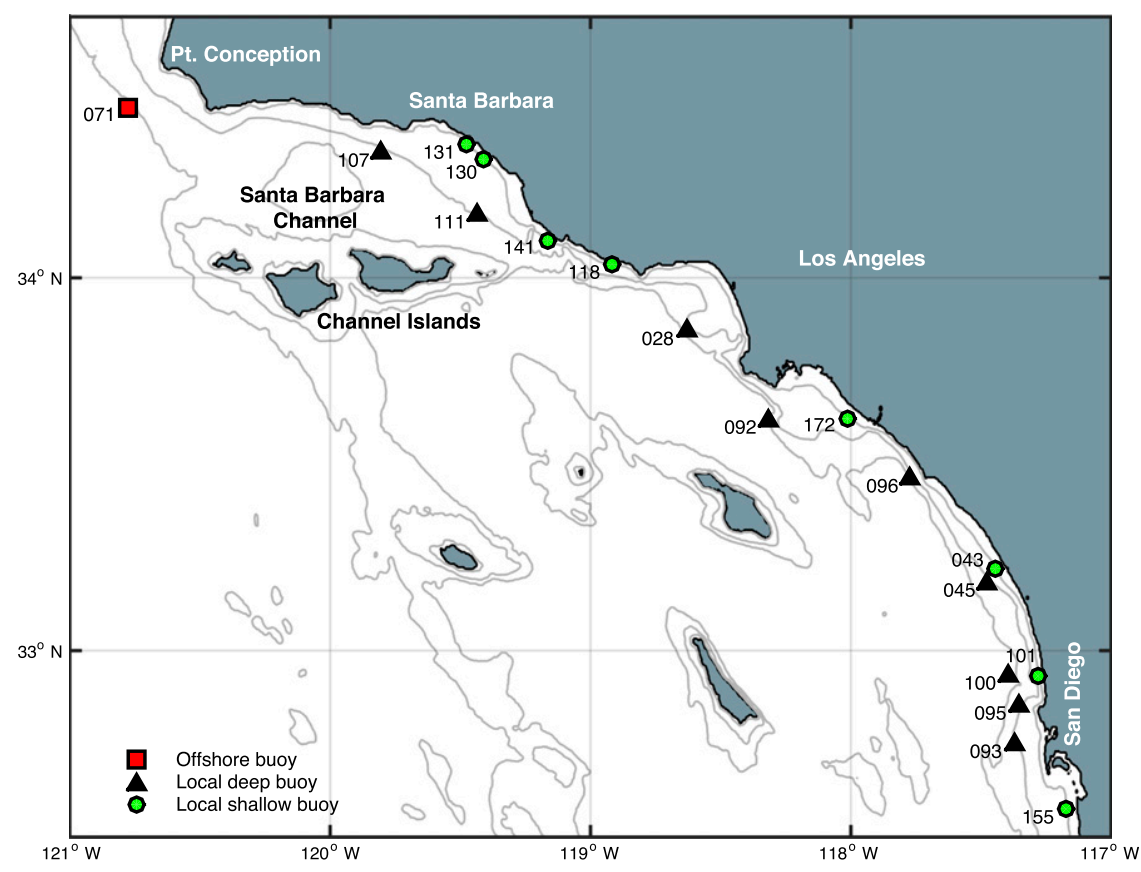

FIG. 1. Southern California map showing offshore buoy (071, square) in 550-m depth used to initialize regional model, local deep validation buoys (black triangles) in depths $100-500 \mathrm{~m}$, and local shallow validation buoys (circles) in 20-m depth. Gray contours show 20-, 100-, and 500-m isobaths.

In some regions for some frequencies, for example, swell energy on the U.S. West Coast, refraction and shoaling dominate the wave field, and nonlinear interactions and energy sources/sinks can be ignored (O'Reilly and Guza 1993; O'Reilly et al. 2016). High spatial, directional, and temporal resolution at small computational costs can be achieved by using raytracing methods to linearly propagate offshore energy inshore (Dorrestein 1960; Longuet-Higgins 1957). Here, we use these linear energy propagation techniques for swell band waves $(0.04-0.09 \mathrm{~Hz})$ in $\mathrm{SC}$, modeling $\sim 60 \%$ of the total shoreward wave energy flux (Fig. 2). Swell model prediction skill at nearshore buoy sites is compared for different offshore boundary condition parameterizations.

In many populated regions, both offshore GWM predictions and buoy observations are available to parameterize a regional wave model boundary. These two data sources are generally independent since operational GWMs do not yet assimilate local buoy observations, though data assimilation is an active area of research (Orzech et al. 2013; Panteleev et al. 2015). When local wave observations are sporadically available (typically in the case of satellite altimetry or short-term buoy observations), biases can be removed from continuous GWM predictions (Mínguez et al. 2011), and the calibrated predictions are then used for the model boundary condition.
However, when long-term offshore wave buoy records are available, it is not clear how to best specify the model boundary condition. GWM predictions have skill, but they still suffer from errors and biases. Additionally, GWM predictions are not typically available in complete frequency-directional detail at all model grid points. In contrast, buoy observations provide an accurate energy distribution in frequency, but they have significant directional ambiguity (Ochoa and Delgado-González 1990). Rogers et al. (2007) created an operational wave model for SC using offshore buoy observations for some model boundaries and GWM predictions for others. In their discussion they experimented with a combination of offshore GWM directional predictions and nondirectional (e.g., energy only) buoy observations, but they found that the combination could increase nearshore prediction errors during bimodal (northern and southern) conditions, common in SC. Here, we extend the preliminary discussion in Rogers et al. (2007), and consider in detail the skill of using GWM and buoy observations in the boundary condition for our SC swell wave model. We examine the predictive skill for significant wave height, wave direction, and alongshore radiation stress with different boundary forcings. We find that buoy observations provide more accurate boundary condition information than GWM predictions and that surprisingly, combinations of GWM and buoy observations are no more skillful than buoy 

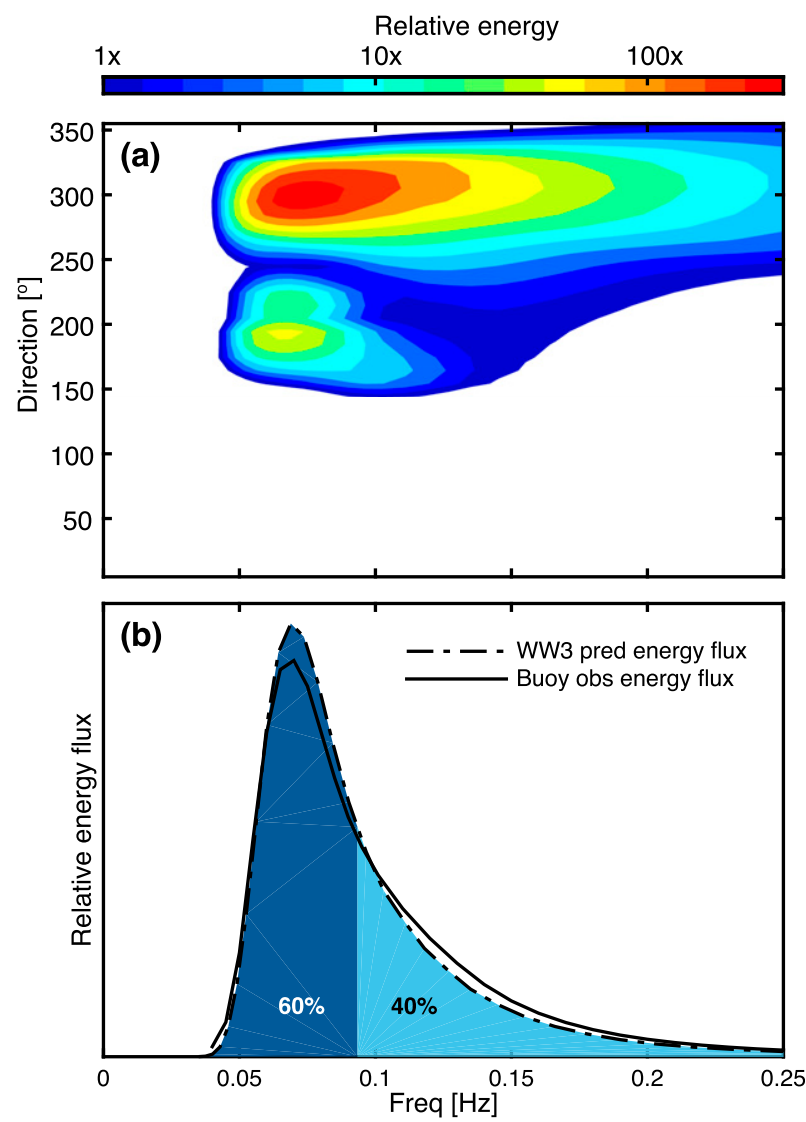

FIG. 2. The 10-yr mean wave conditions at offshore buoy 071 . (a) Mean GWM (NOAA-WW3) wave energy predictions (color) vs frequency and direction. (b) GWM- (dashed) and buoy-measured (solid) mean energy flux $\left(E c_{g}\right)$ vs frequency (integrated over direction). Swell $(0.04-0.09 \mathrm{~Hz})$ and sea $(0.09-0.25 \mathrm{~Hz})$ frequency bands account for $60 \%$ and $40 \%$ of the offshore energy flux, respectively.

observations alone. While our study is limited to remotely generated swell wave energy, future work will consider the more difficult question of using GWM and buoy observations for the boundary condition at higherfrequency wave energy, where local wind generation may be important.

Section 2 discusses the limitations of directional wave buoy observations and various methods typically used to construct directional spectra. Additionally, formulations for alongshore radiation stress and alongshore transport are reviewed. Section 3 describes the study region, buoy observations, wave model methodology, and boundary condition methods tested. Section 4 compares the skill of different boundary conditions. Section 5 discusses regional model error, examines the effect of small directional biases in the context of alongshore transport, and considers the effect of weak coastal reflection.

\section{Background}

Phase-averaged offshore wave energy $F(f, \theta)$ is decomposed into the total energy $E(f)$ at each frequency band $f$ and a normalized directional distribution $D(f, \theta)$ such that

$$
F(f, \theta)=E(f) D(f, \theta) .
$$

Using accelerometers or onboard GPS, directional wave buoys measure vertical and horizontal surface translations. Once transformed into frequency space, combinations of co- and quadspectra provide estimates of $E(f)$ and four integral constraints on $D(f, \theta)$ (LonguetHiggins et al. 1963; Long 1980). Dropping $f$ for clarity, the directional constraints in matrix notation are

$$
\mathbf{d}=\left[\begin{array}{l}
a_{1} \\
b_{1} \\
a_{2} \\
b_{2}
\end{array}\right]=\left[\begin{array}{l}
\int D(\theta) \cos \theta d \theta \\
\int D(\theta) \sin \theta d \theta \\
\int D(\theta) \cos 2 \theta d \theta \\
\int D(\theta) \sin 2 \theta d \theta
\end{array}\right] .
$$

Additionally, normalization yields

$$
\int D(\theta) d \theta=1
$$

and the energy cannot be negative,

$$
D(\theta) \geq 0 \text {. }
$$

The directional constraints on $D(\theta)$ in (2), often referred to as low-order moments, can be used to estimate the bulk directional properties: mean direction $(\theta)$, spread $(\sigma)$, skewness, and kurtosis. Additionally, first $\left(a_{1}, b_{1}\right)$ and second $\left(a_{2}, b_{2}\right)$ low-order moments can be used independently to estimate $\theta$ and $\sigma$ such that

$$
\begin{aligned}
\theta_{1} & =\arctan \left(b_{1} / a_{1}\right), \\
\theta_{2} & =2 \arctan \left(b_{2} / a_{2}\right), \\
\sigma_{1} & =\sqrt{2\left[1-\left(a_{1}^{2}+b_{1}^{2}\right)^{1 / 2}\right],} \\
\sigma_{2} & =\sqrt{\frac{1}{2}\left[1-\left(a_{2}^{2}+b_{2}^{2}\right)^{1 / 2}\right]}
\end{aligned}
$$

(Kuik et al. 1988; Herbers et al. 1999, 2012). The $180^{\circ}$ ambiguity in $\theta_{2}$ does not differentiate between normally incident waves and their specular reflection. The corresponding $\sigma_{2}$ estimate is advantageous when the properties of incoming waves only are sought, whereas $\sigma_{1}$ is a sensitive 

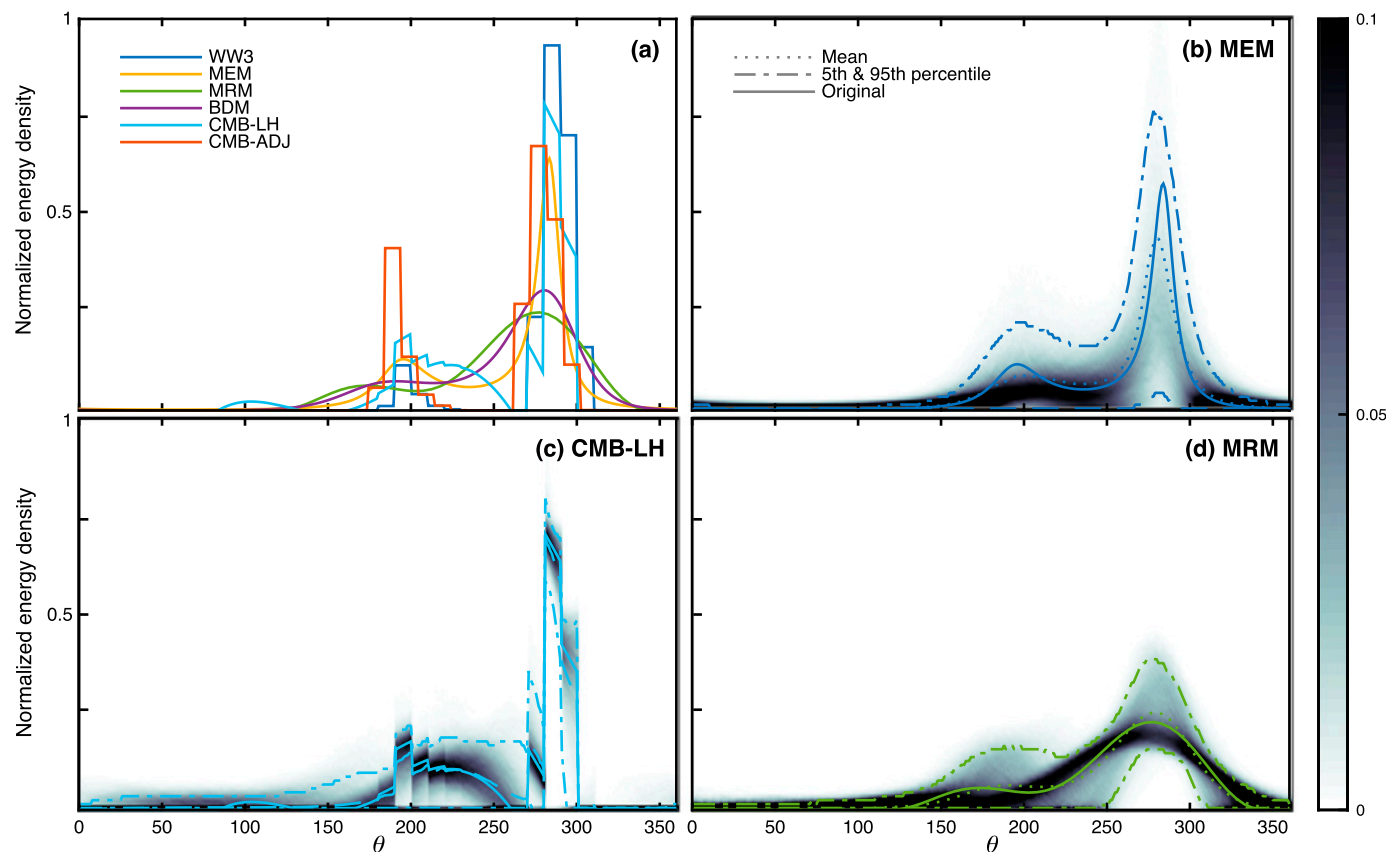

FIG. 3. (a) Normalized (to unit area) energy density vs direction for a typical swell frequency band, at offshore buoy 071. Methods (see legend) are buoy only (MEM, MRM, BDM), model only (WW3), and combinations (CMB-LH, CMB-ADJ). Probability distributions (likelihood gray scale on right) for (b) MEM, (c) CMB-LH, and (d) MRM for the expected uncertainty in a $1-\mathrm{h}$ record [degree of freedom $(\mathrm{DOF})=64$ ]. Solid colored curves repeat curves in (a). Mean and scatter [5\% and $95 \%$ levels; see legend in (b)] are also shown, respectively.

reflection detector (Herbers et al. 1999). Statistics of directional moments and bulk properties for a finite-length buoy record are in Long (1980) and Kuik et al. (1988).

Low-order moments are accurately measured by a Datawell directional wave buoy (O'Reilly et al. 1996), but they do not define a unique directional spectrum (Ochoa and Delgado-González 1990), and a continuous or finely discretized $D(\theta)$ is needed at wave model boundaries. Estimating $D(\theta)$ from limited information in (2), (3), and (4) is a classic geophysical inverse problem (Backus and Gilbert 1967) requiring an additional subjective constraint. Minimum roughness $\left(d^{2} D / d \theta^{2}\right)$ yields the simplest solution consistent with the data and is often used (Constable et al. 1987). In the last four decades, various constraints have been used to find $D(\theta)$ given observed buoy moments and constraints [(3), (4)], including maximum entropy (Kobune and Hashimoto 1985; Lygre and Krogstad 1986), minimum roughness (Herbers and Guza 1990), and others (Oltman-Shay and Guza 1984; Hashimoto and Konbune 1988). These methods optimize different flavors of smoothness, and the ability of each constraint to accurately represent the true directional spectrum likely varies regionally and seasonally. All smoothness constraints necessarily fail when the true $D(\theta)$ is complex owing to multiple directional sources. As an alternative to smoothness, Long and Hasselmann (1979) presented a method to find the $D(\theta)$ that is consistent, in a least squares sense, with both measured buoy moments and an a priori-preferred spectrum (e.g., generated by a GWM).

The uncertainty inherent in buoy observations is illustrated in Fig. 3a; the same buoy observations [(2)], typical of bimodal directional spectra in SC, are input into the maximum entropy method (MEM; Lygre and Krogstad 1986), minimum roughness method (MRM; Herbers and Guza 1990), and the Bayesian direct method $^{1}$ (BDM; Hashimoto and Konbune 1988). Although the direction of the primary northwest peak is fairly consistent, the estimators disagree on the amplitudes and widths of the two peaks even though each of these distributions are consistent with the same deterministic low-order moments. Furthermore, each of these estimates varies considerably owing to statistical fluctuations of $\mathbf{d}$ in (2). For a typical 1-h buoy record, known uncertainty in the low-order moments (Long

\footnotetext{
${ }^{1}$ The method is often unstable for point-source wave observations, for example, buoy or collocated pressure gauge and current meters (PUV); however, we ignore the Akaike information criterion (AIC) and seek stable spectra with the closest fit to the observed low-order moments.
} 
$1980)$ is used to generate many $(N=10000)$ random realizations of $\mathbf{d}$. MEM and MRM are then used to create many realizations of $D(\theta)$ from which a probability distribution is created, illustrating the statistical uncertainty (Figs. 3b,d). Figure 3c shows a similarly derived distribution for a method combing buoy observations and GWM predictions introduced in section 3d. A 3-h averaging of buoy observations is used in our analysis to reduce the statistical blurring of $D(\theta)$.

Intuition about the fundamental limitations of buoy observations follows from considering the number of system knowns and unknowns. Buoy observations provide five knowns [(2), (3)]. A unimodal system can be described by a mean direction, amplitude, directional spread, skewness, and kurtosis (five unknowns). A bimodal system can be described with two peak amplitudes, two peak directions, and with one known left to describe (poorly) both peak widths. The properties of two individual peaks are blurry, and with more than two peaks, buoy moments cannot even describe peak locations and amplitudes.

Despite these inherent limitations, directional buoys measure the alongshore radiation stress $S_{x y}$ exactly, without the need to estimate $D(f, \theta)$ (Longuet-Higgins and Stewart 1964). The term $S_{x y}$ is directly proportional to the low-order moment $b_{2}^{(r)}$, where

$$
\begin{aligned}
S_{x y} & =\iint\left(\frac{c_{g}}{c}\right) E(f) D(f, \theta) \sin \theta \cos \theta d \theta d f, \\
& =\frac{1}{2} \int\left(\frac{c_{g}}{c}\right) E(f) b_{2}^{(r)}(f) d f,
\end{aligned}
$$

and $b_{2}^{(r)}$ is the buoy moment rotated into the local shorenormal reference frame. The term $S_{x y}$ is an important driver of alongshore currents (Guza et al. 1986) and transport (Komar and Inman 1970). In the frequently used Coastal Engineering Research Center (CERC) transport formula (Komar and Inman 1970), the immersed weight of sediment $I$ is

$$
I=\left.K E c_{g} \frac{\sin 2 \theta}{2}\right|_{\text {breaking }}=\left.K S_{x y} c_{p}\right|_{\text {breaking }},
$$

where $c_{g}$ is group velocity, $c_{p}$ is phase velocity, and $K$ is an empirical constant typically $0.4-0.8$. The simplistic CERC formula performs similarly to sophisticated process models (Haas and Hanes 2004).

\section{Methods}

\section{a. Study region}

Southern California (SC) extends from Point Conception to the United States-Mexico border (Fig. 1), and is exposed to distant North and South Pacific storms as well as local wind swell (Adams et al. 2008). Complex coastal bathymetry and shadowing from offshore islands create a spatially variable nearshore wave field that is extremely sensitive to the offshore wave direction. Directional inaccuracies of the waves specified at the offshore boundary are believed to be the dominant source of wave model prediction error in Southern California (O'Reilly and Guza 1993; Rogers et al. 2007).

\section{b. Swell wave model}

Modern third-generation wave models [e.g., WaveWatch III (WW3) and Simulating Waves Nearshore (SWAN)] solve the spectral wave action equation (Hayes 1970), which includes refraction, shoaling, wind generation, nonlinear wave interactions, wave-current interactions, whitecapping, bottom dissipation, and depth-limited breaking. These commonly used state-ofthe-art models are computationally expensive with high spatial resolution and long duration. In SC, the transformation of low-frequency $(0.04-0.09 \mathrm{~Hz})$ swell is dominated by island sheltering, and bathymetrycontrolled shoaling and refraction (O'Reilly and Guza 1993; O'Reilly et al. 2016). Our SC swell wave model, limited to $0.04-0.09-\mathrm{Hz}$ frequencies, captures these effects using frequency-direction-dependent transfer coefficients derived from backward ray-tracing methods (see appendix A; Longuet-Higgins 1957; Dorrestein 1960; Mehaute and Wang 1982). The transfer coefficients represent a linear relation between the offshore and nearshore wave field. Because energy travel time across SC can be significant, 10+ hours, frequencydirection-dependent time lags are included by assuming energy propagation at the theoretical group velocity (Fig. A1), following O'Reilly and Guza (1998) and O'Reilly et al. (2016). This methodology significantly reduces computation expense, but it requires the assumption that offshore wave conditions are spatially homogenous (with time lags) in the along-crest direction (Fig. A1). This contrasts with typical modern wave models that accept heterogeneous boundary conditions offshore. However, offshore GWM predictions confirm along-crest spatial homogeneity over the few $100-\mathrm{km}$ scales of SC for the remotely generated waves that dominate the swell band $(<0.09 \mathrm{~Hz})$; details are in appendix A. Our swell model predictions are therefore a linear function of offshore energy $E(f, \theta)$ with an appropriate time lag $\tau(f, \theta)$.

The swell model captures approximately $60 \%$ of offshore energy flux (Fig. 2b). Model transfer coefficients are created at a $0.005-\mathrm{Hz}$ frequency resolution, typical of buoy hourly records, and $1^{\circ}$ directional resolution that is 
TABLE 1. Wave buoy names, depths, and site occupation dates.

\begin{tabular}{|c|c|c|c|c|c|}
\hline NOAA & CDIP & Name & Depth (m) & Start & End \\
\hline \multicolumn{6}{|c|}{ Deep } \\
\hline 46218 & 071 & Harvest & 549 & $2000 \mathrm{Jan}$ & $2009 \mathrm{Dec}$ \\
\hline 46216 & 107 & Goleta & 182 & 2002 Jun & $2009 \mathrm{Dec}$ \\
\hline 46217 & 111 & Anacapa & 114 & 2002 Jun & 2009 Dec \\
\hline 46221 & 028 & Santa Monica & 363 & $2000 \mathrm{Mar}$ & $2009 \mathrm{Dec}$ \\
\hline 46222 & 092 & San Pedro & 457 & $2000 \mathrm{Jan}$ & $2009 \mathrm{Dec}$ \\
\hline 46223 & 096 & Dana Point & 373 & $2000 \mathrm{Jul}$ & 2009 Dec \\
\hline 46224 & 045 & Oceanside & 220 & 2000 Jan & 2009 Dec \\
\hline 46225 & 100 & Torrey Pines & 549 & 2001 Jan & $2009 \mathrm{Dec}$ \\
\hline 46231 & 093 & Mission Bay & 201 & 2005 Oct & $2009 \mathrm{Dec}$ \\
\hline 46226 & 095 & Point La Jolla & 181 & 2000 Jan & 2005 Oct \\
\hline \multicolumn{6}{|c|}{ Shallow } \\
\hline - & 131 & Rincon Point & 21 & 2005 Sep & 2007 Apr \\
\hline 46228 & 130 & Pitas Point & 20 & 2004 Oct & 2005 Sep \\
\hline 46234 & 141 & Port Hueneme & 21 & 2007 Apr & $2009 \mathrm{Feb}$ \\
\hline - & 118 & Leo Carillo & 20 & $2003 \mathrm{Apr}$ & 2004 Mar \\
\hline 46230 & 172 & Huntington Beach & 22 & 2005 Jun & 2006 Nov \\
\hline 46242 & 043 & Camp Pendleton & 20 & 2008 Jan & $2009 \mathrm{Dec}$ \\
\hline 46235 & 155 & Imperial Beach & 18 & $2006 \mathrm{Dec}$ & $2009 \mathrm{Dec}$ \\
\hline - & 101 & Torrey Pines Inner & 20 & $2001 \mathrm{Apr}$ & 2004 Mar \\
\hline
\end{tabular}

higher than commonly used but desired for directionally sensitive SC. The linear framework allowed for relatively rapid testing of different boundary conditions from 2000 to 2009. All wave properties discussed in the following are swell band averaged such that some property $\bar{x}$ is

$$
\bar{x}=\frac{\int_{0.04}^{0.09} E(f) x(f) d f}{\int_{0.04}^{0.09} E(f) d f} .
$$

\section{c. Buoy observations}

Quality-controlled, hourly, Datawell directional buoy observations (energy and low-order directional moments) from the Coastal Data Information Program (CDIP; http://cdip.ucsd.edu/) were smoothed with a 3-h running mean. The reduction of statistical uncertainty with 3-h smoothing is particularly important for lowfrequency narrow-banded swell, where the effective degrees of freedom (EDOF) are low (Elgar 1987). In extreme cases (e.g., 20-s southwest swell), the EDOF of a 1-h buoy record can be less than 100 for the entire swell band spectrum, corresponding to approximately a $\pm 25 \%$ uncertainty (90th percentile) in measured energy. Swell conditions are often stationary for $3 \mathrm{~h}$, and minimal temporal resolution is lost. The regional wave model is initialized with the exposed offshore buoy, 071, in 550-m water depth. The model is tested with nine coastal deep-water buoys in depths ranging from 180 to $500 \mathrm{~m}$, and eight local shallow-water buoys in 20-m depth (Fig. 1). Buoy site occupation duration varied (Table 1 ). Shallow buoys were typically deployed for the shortest times, approximately one year.

\section{d. Boundary conditions}

Estimates of $F(f, \theta, t)$ offshore were specified from buoy 071 observations and from GWM predictions. Buoy 071 has wide directional exposure and a 10-yr time series of energy $E_{b}(f)$ and directional moments $\mathbf{d}_{b}(f)$. For hindcast GWM predictions, we use the National Oceanic and Atmospheric Administration (NOAA) WW3 reanalysis (Tolman 2009; Chawla et al 2011, 2013). The U.S. West Coast reanalysis has a 4-arc-min $(7 \mathrm{~km})$ spatial resolution, 3-h temporal resolution, $10^{\circ}$ directional resolution, and logarithmic frequency spacing. Complete WW3 energy-directional spectra predictions, $F_{\mathrm{Ww} 3}(f, \theta, t)$, and collocated buoy 071 observations from 2000 to 2009 are used to specify boundary conditions. WW3 energy $F_{\mathrm{Ww} 3}(f, \theta, t)$ is rebanded in frequency to match typical buoy spectral output $(0.005-\mathrm{Hz}$ bandwidth from 0.04 to $0.09 \mathrm{~Hz})$ and interpolated by nearest neighbor to 1 -h temporal and $1^{\circ}$ directional resolution. Excluded are the less-than-6months' total when buoy 071 observations were 
unavailable. Boundary conditions tested are divided into three categories:

- GWM only: $F_{\mathrm{WW} 3}(f, \theta)$ is used exclusively, hereafter WW3.

- Buoy only: $F(f, \theta)$ is created with buoy 071 observations. Three different methods-MEM, MRM, and BDM (see section 2)-are tested to estimate the discrete directional spectrum from directional buoy observations [(2)].

- Combined (CMB): Buoy 071 observations are combined with GWM predictions of the normalized directional distribution $D_{\mathrm{ww} 3}(f, \theta)$ in three different ways.

The first CMB method, CMB-E, multiplies the buoy $E_{b}$ by $D_{\mathrm{ww} 3}$, after the discussion of Rogers et al. (2007). Buoy directional information is not used. The second CMB method, CMB-LH, combines $D_{\text {ww3 }}$ with both $E_{b}$ and $\mathbf{d}_{b}$ (Long and Hasselmann 1979). The optimal directional spectrum $D_{\mathrm{LH}}$ minimizes

$$
\int\left(D_{\mathrm{LH}}-D_{\mathrm{WW} 3}\right)^{2} d \theta
$$

while satisfying $\mathbf{d}_{b}[(2)]$ and constraints (3) and (4). The computationally efficient iterative method developed by Long and Hasselmann (1979) terminates when constraints are met within the expected statistical uncertainty (Lawson and Long 1983; Long 1980). We tested this iterative method and found that fitting constraints exactly yielded unbiased spectra with comparatively better nearshore predictive skill. Additionally, we compared the iterative method solutions to those from a modern optimization numerical solver, CVX, a package for specifying and solving convex problems [CVX (MATLAB), CVXPY (Python), and Convex.j1 (Julia); Boyd and Vandenberghe 2004; Grant and Boyd 2013]. Both solutions were robust for most conditions; however, the iterative method occasionally failed to converge for very low-energy spectra. We ultimately used CVX to solve the minimization; however, we continue to refer to the solution as CMB-LH. Once found, $D_{\mathrm{LH}}$ is multiplied by $E_{b}$ to construct $F(f, \theta)$, as in the CMB-E method.

The third CMB method, CMB-ADJ, is not formally optimal but relies instead on physically meaningful adjustments to $D_{\mathrm{ww} 3}$, including a series of shifts and smoothings to minimize misfit to constraints [(2)] with $\mathbf{d}_{b}$. Initial testing showed the ideal simultaneous optimization was computationally burdensome and provided marginal improvement over the simpler sequential adjustment process used below at each frequency bin for each hour record.
1) Time lag/leads in $F_{\mathrm{Ww} 3}(f, \theta)$ are corrected for each 1-h time step by considering the optimal time lag correlation between GWM predicted energy, $\int F_{\mathrm{Ww} 3}(f, \theta) d \theta$, and $E_{b}$ inside a \pm 36 -h window. Typically, time lag/lead corrections were small $(<3 \mathrm{~h})$ and consistent across wave events.

2) Significant peaks in $D_{\text {ww3 }}$ are found (using MATLAB's Signal Processing toolbox) that meet the following criteria:

(i) Each peak contains at least $2 \%$ of primary peak energy.

(ii) Peaks spaced less than $55^{\circ}$ are considered a single peak.

3) If $D_{\mathrm{ww} 3}$ is unimodal ( $49 \%$ of records), then

(i) Rotate $D_{\mathrm{Ww} 3}$ to match $\theta_{1}$ measured by buoy 071 .

(ii) Estimate $\sigma_{2}$ for $D_{\mathrm{Ww} 3}$ from (2) and (5). Smooth $D_{\mathrm{Ww} 3}$ by convolving with a simple boxcar repeatedly until $\sigma_{2}$ for $D_{\mathrm{Ww} 3}$ is approximately equal to $\sigma_{2}$ measured by buoy 071 . Here, $\sigma_{2}$ is used to sometimes avoid oversmoothing due to buoy-observed coastal reflection.

4) If $D_{\mathrm{ww} 3}$ is bimodal ( $49 \%$ of records), then

(i) Use brute force to find the shift of energy between the two $D_{\mathrm{ww} 3}$ peaks such that the misfit to $\mathbf{d}_{b}$, weighted by the uncertainty in $\mathbf{d}_{b}$ (Long 1980), is minimized.

(ii) Simultaneously and independently shift both peak directions (with a maximum shift of $25^{\circ}$ ) to minimize misfit to $\mathbf{d}_{b}$.

$5)$ If $D_{\mathrm{wW} 3}$ contains three or more peaks $(2 \%$ of records), then make no directional adjustments to the WW3 spectrum as buoy directional information is too limited.

Last, as in the previous methods, the adjusted $D_{\mathrm{ww} 3}$ is multiplied by $E_{b}$ to form $F(f, \theta)$. These adjustments to $D_{\text {ww3 }}$ include some threshold values that may be region specific. Additionally, the performance of the methods is dependent on the accuracy of the GWM; statistical chatter in buoy observations can only degrade the predictions of a theoretically error-free GWM. However, here we show that buoy-based adjustments with these thresholds do detectably improve the GWM-only, WW3, and boundary condition method skill.

\section{Results}

\section{a. Significant wave height, direction, and spread}

Model predictions (using the boundary conditions described above) are compared with observations at sheltered deep buoys using standard metrics [root-mean-square error (RMSE), bias, model skill $R^{2}$, and squared correlation 
TABLE 2. Mean model skill at local deep buoys.

\begin{tabular}{lccccccccccrrr}
\hline \hline \multicolumn{1}{c}{ Model } & $\begin{array}{c}H_{\text {sig }} \\
\text { Mean }\end{array}$ & RMSE & $\mid$ Bias $\mid$ & $R^{2}$ & $r^{2}$ & $\begin{array}{c}\overline{\theta_{1}} \\
\text { RMSE }\end{array}$ & $\mid$ Bias $\mid$ & $R^{2}$ & $r^{2}$ & $\begin{array}{c}\overline{\sigma_{1}} \\
\text { RMSE }\end{array}$ & Bias & $R^{2}$ & $r^{2}$ \\
\hline & $(\mathrm{m})$ & $(\mathrm{m})$ & $(\mathrm{m})$ & - & - & $\left(^{\circ}\right)$ & $\left.{ }^{\circ}\right)$ & - & - & $\left(^{\circ}\right)$ & $\left(^{\circ}\right)$ & - \\
WW3 & 0.65 & 0.21 & 0.09 & 0.53 & 0.73 & 15 & 4 & 0.72 & 0.77 & 14 & -11 & -1.53 & 0.41 \\
CMB-E & 0.52 & 0.15 & 0.06 & 0.56 & 0.67 & 13 & 4 & 0.64 & 0.69 & 13 & -10 & -1.61 & 0.34 \\
CMB-LH & 0.51 & 0.13 & 0.05 & 0.60 & 0.71 & 13 & 3 & 0.65 & 0.68 & 11 & -9 & -1.16 & 0.33 \\
CMB-ADJ & 0.52 & 0.13 & 0.03 & 0.63 & 0.72 & 11 & 2 & 0.72 & 0.74 & 11 & -9 & -1.06 & 0.42 \\
MEM & 0.51 & 0.13 & 0.04 & 0.63 & 0.72 & 11 & 3 & 0.69 & 0.76 & 10 & -8 & -0.55 & 0.45 \\
MRM & 0.52 & 0.12 & 0.02 & 0.66 & 0.73 & 13 & 7 & 0.62 & 0.74 & 11 & -9 & -1.16 & 0.37 \\
BDM & 0.51 & 0.12 & 0.03 & 0.66 & 0.74 & 11 & 5 & 0.69 & 0.77 & 10 & -8 & -0.56 & 0.47 \\
\hline
\end{tabular}

$r^{2}$; see appendix B for definitions]. Model skill varies across boundary condition, buoy validation site, and metric. Comprehensive skill results for significant wave height $H_{\text {sig }}$, mean direction $\theta_{1}$, and directional spread $\sigma_{1}$ for all boundary conditions at all buoy validation sites are in Tables S1 and S2 in the online supplementary material. Simple averages at deep buoy validation sites (Table 2) are used to rank boundary condition skill in Table 3, where the lowest score is best. Bias magnitude was averaged for $H_{\text {sig }}$ and $\theta_{1}$ to avoid canceling errors. CMB-ADJ and MEM have the highest $\theta_{1}$ skill, while MRM and BDM have the highest $H_{\text {sig }}$ skill. WW3 has overall the lowest skill followed by CMB-E. CMB-ADJ has the highest overall skill followed by BDM. Models using buoy observations in the boundary condition (buoy only and CMB) outperform WW3 alone. However, directional bias is lowest for WW3 and CMB boundary conditions at local deep buoys. Bias in $\sigma_{1}$ predictions is negative for all boundary conditions and largest at low frequencies (not shown), suggesting weak coastal reflection (section 5c).

The spatial distribution of model skill for a subset of boundary conditions shows that WW3 initialization consistently has relatively large RMSE and bias; in Fig. 4 the upper-left quadrant is large and dark at most buoys, for both $H_{\text {sig }}$ and $\theta_{1}$. The term $H_{\text {sig }}$ from WW3 is biased high at the initialization offshore buoy 071 $(+23 \mathrm{~cm})$, and WW3 is correspondingly high in the southern half of the model domain. In contrast, WW3 initializations are biased low for $H_{\text {sig }}$ in the Santa Barbara Channel (SBC), closest to the initialization buoy (Fig. 4a), and directional errors and biases are also large. Boundary condition MRM shows particularly large positive (northward) directional bias in the southern end of the SBC. A ranking of buoy site skill, averaged across select boundary conditions (Table 4), indicates the poorest model performance at buoy site 107 (in SBC) and at the southernmost buoy site, 093 .

\section{b. Alongshore radiation stress}

Mean (time averaged) $S_{x y}$ model predictions and shallow (20-m depth) buoy observations are compared in the local shore-normal reference frame estimated from depth contours onshore of each buoy; positive $S_{x y}$ indicates northward up-coast transport (Fig. 5). Modeled and observed $S_{x y}$ usually agree, at least qualitatively. Boundary conditions using offshore buoy observations generally outperform WW3 alone. No buoy-based method clearly performs best. Uncertainty bars on $S_{x y}$ correspond to $\pm 2.5^{\circ}$ shifts of the local shore normal. At buoy 155 , mean $S_{x y}$ is near zero, and the results are relatively sensitive to the definition of beach normal. At buoy 043 (Oceanside Harbor), different offshore boundary conditions yield the widest range of $S_{x y}$ with WW3 biased high and MRM biased low.

Depth contours offshore of Oceanside Harbor (gray, Fig. 6a) were used to estimate local shore normals (dark magenta, Fig. 6a) that were alongshore averaged (excluding the harbor area) to avoid the influence of slight shore-normal changes on $S_{x y}$. Predicted $H_{\text {sig }}, \theta_{2}$, and $S_{x y}$ on the 10-m-depth contour (bright green) show similar alongshore variation about constant offsets (Figs. 6b-d). WW3 predicts the highest wave heights and the most oblique waves, resulting in the highest $S_{x y}$. These are compared to buoy 043 observations of $S_{x y}$ (Fig. 6d; $S_{x y}$

TABLE 3. Model skill rankings averaged across deep local buoys for various boundary conditions and select metrics (low ranking indicates higher skill).

\begin{tabular}{lcccccc}
\hline \hline $\begin{array}{l}\text { Boundary } \\
\text { Condition }\end{array}$ & $\begin{array}{c}H_{\text {sig }} \\
R^{2}\end{array}$ & $\mid$ Bias $\mid$ & $\begin{array}{c}\overline{\theta_{1}} \\
R^{2}\end{array}$ & $\mid$ Bias $\mid$ & Total & Rank \\
\hline WW3 & 7 & 7 & 6 & 4 & 24 & 7 \\
CMB-E & 6 & 6 & 5 & 5 & 22 & 6 \\
CMB-LH & 5 & 5 & 4 & 2 & 16 & 4 \\
CMB-ADJ & 3 & 3 & 1 & 1 & 8 & 1 \\
MEM & 4 & 4 & 2 & 3 & 13 & 3 \\
MRM & 2 & 1 & 7 & 7 & 17 & 5 \\
BDM & 1 & 2 & 3 & 6 & 12 & 2 \\
\hline
\end{tabular}


(a) $-30$

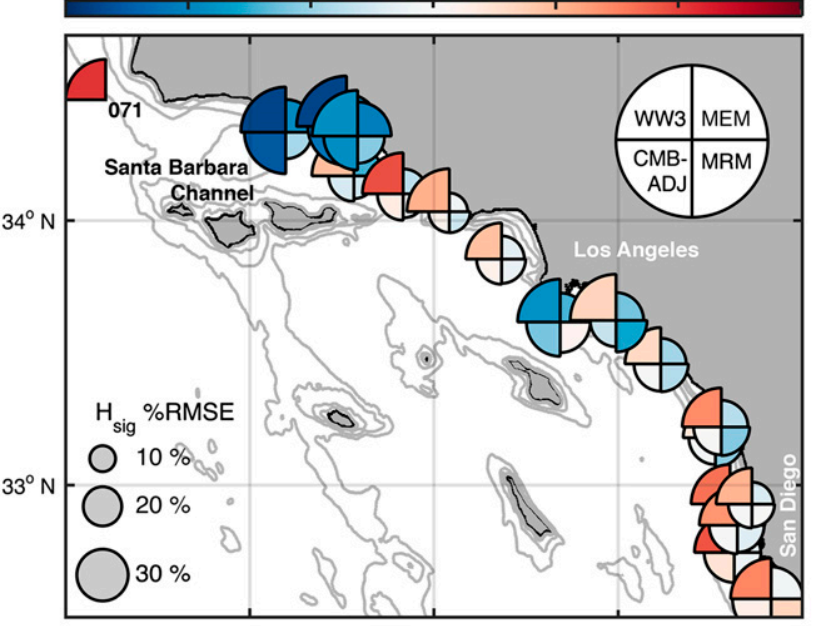

$121^{\circ} \mathrm{W}$
$\mathbf{H}_{\text {sig }}$ Bias [\%] $-20$ $120^{\circ} \mathrm{W}$ (b)

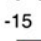

$-15 \quad-10$

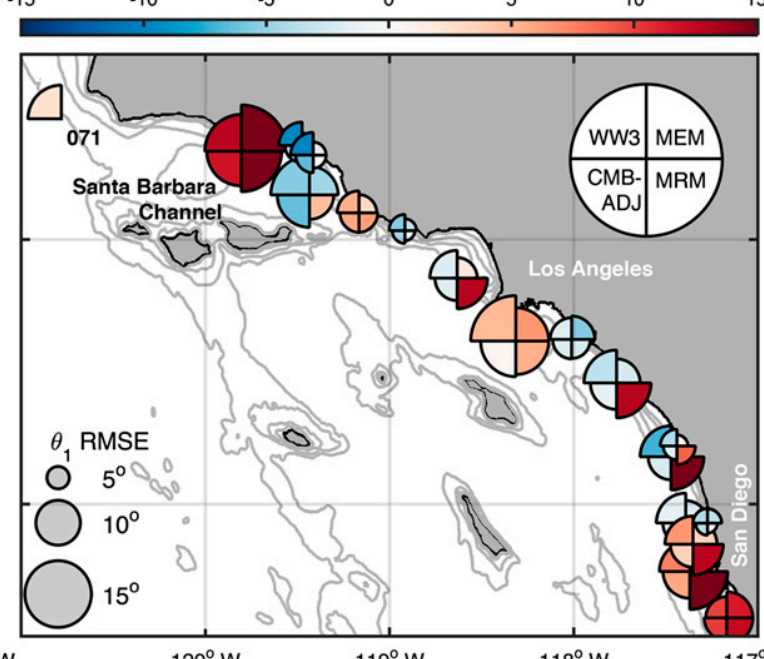

FIG. 4. Model performance at each SC nearshore buoy location for (a) wave height $H_{\text {sig }}$ and (b) wave direction $\theta_{1}$. Each circle quadrant shows a different boundary condition (key, upper right). Circle quadrant size shows RMSE (key, lower left), and color shows bias (color bar, above). Normalized error metrics [\% RMSE and \% bias, in (B1)] are used in (a). A small quadrant indicates small RMSE, and a pale color indicates small bias. GWM (NOAA-WW3) initialization (upper-left quadrant), in all regions and for both $H_{\text {sig }}$ and $\theta_{1}$, is relatively large and dark, indicating relatively high RMSE and bias. Gray contours show 20-, 50-, 100-, and 500-m isobaths.

conserved assuming depth contours are parallel). Alongshore sediment transport estimated using the CERC formula $^{2}[(7)]$ with constant $K=0.6$ (Fig. 6e) yields estimates between 2 and $9 \times 10^{5} \mathrm{~m}^{3} \mathrm{yr}^{-1}$. Shaded regions show transport estimates for a typical range of CERC constant $K(0.4<K<0.8)$, smoothed by 1.5 -km alongshore locally weighted scatterplot smoothing (lowess) filter. Longshore transport gradients, estimated from smoothed transport estimates, are similar for all predictions (Fig. 6f). Note this gradient estimate ignores the effect of the harbor and small-scale $(<1.5 \mathrm{~km})$ alongshore variations in $S_{x y}$.

\section{Discussion}

\section{a. Optimal boundary condition}

Offshore buoy observations improve WW3 nearshore wave prediction skill for most metrics at most buoy validation sites. However, improvement is lowest for CMB-E, where the buoy-measured energy is combined with $D_{\mathrm{ww} 3}$. Model skill further increases when buoy energy and directional information are used, and CMB-ADJ outperforms CMB-LH (Table 3). Detailed examination of CMB-LH reveals that the least squares constraint in (8) often adds spurious directional peaks to

\footnotetext{
${ }^{2}$ Waves were linearly shoaled to breaking $\left(E c_{g}=\right.$ constant $)$ with breaking constant $\gamma=0.7$.
}

the directional distribution. For example in Fig. 3a, CMB-LH creates a third peak at $100^{\circ}$ and adds energy in the gap between the peaks. In contrast, CMB-ADJ more physically adjusts peak amplitude, direction, and width. While mathematically convenient and theoretically elegant, the CMB-LH squared difference constraint is not effective. CMB-ADJ, though crude, outperforms CMB-LH.

While buoy observations improved the WW3 boundary condition, the skill of CMB-ADJ is similar to buoyonly methods; CMB-ADJ $H_{\text {sig }}$ skill is lower, but the direction skill is higher, yielding an overall higher skill ranking (Table 3). Given the directional ambiguity in buoy observations (e.g., Fig. 3), we expected that offshore

TABLE 4. Mean skill for best models (CMB-ADJ, MEM, BDM) ranked across local deep buoys for select metrics (low ranking indicates higher skill). Buoys are listed from north to south.

\begin{tabular}{ccccccc}
\hline \hline & \multicolumn{7}{c}{$H_{\text {sig }}$} & & $\overline{\theta_{1}}$ & \\
NOAA & CDIP & $R^{2}$ & $\mid$ Bias $\mid$ & $R^{2}$ & $\mid$ Bias $\mid$ & Total \\
\hline 46216 & 107 & 1 & 9 & 9 & 9 & 28 \\
46217 & 111 & 4 & 8 & 1 & 5 & 18 \\
46221 & 028 & 2 & 2 & 5 & 4 & 13 \\
46222 & 092 & 3 & 7 & 4 & 6 & 20 \\
46223 & 096 & 7 & 6 & 3 & 3 & 19 \\
46224 & 045 & 9 & 5 & 6 & 1 & 21 \\
46225 & 100 & 6 & 4 & 2 & 2 & 14 \\
46231 & 093 & 8 & 3 & 8 & 8 & 27 \\
46226 & 095 & 5 & 1 & 7 & 7 & 20 \\
\hline
\end{tabular}




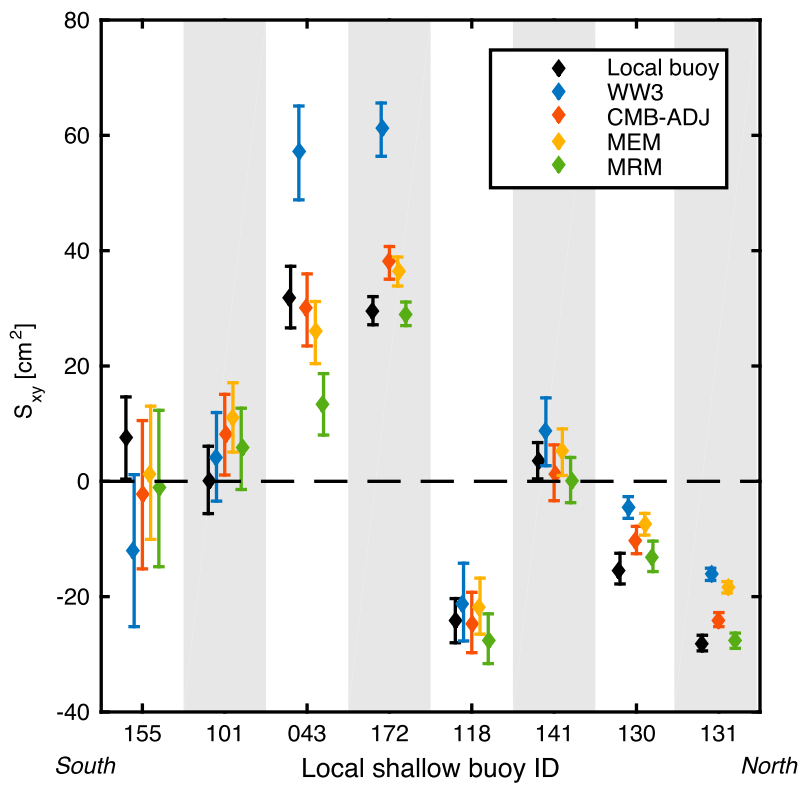

FIG. 5. Observed (black, local buoy observations) and predicted (using methods in legend) mean $S_{x y}$ at local shallow (20-m depth) buoy sites (arranged south to north on the $x$ axis). Positive $S_{x y}$ values indicate northward up-coast transport. Bars indicate $S_{x y}$ range for $\pm 2.5^{\circ}$ local shore-normal rotation. Predictions are offset horizontally for clarity.

GWM directional spectra predictions offshore, informed by global wind fields and sophisticated model physics, would provide a powerful additional constraint. However, we were surprised to find that our combinations of offshore GWM spectra and buoy observations in the offshore boundary condition parameterization were similarly skilled to buoy-only methods. We show below that CMB-ADJ and buoyonly methods have similar skill because details in GWM directional spectra are often well represented by the first four low-order moments [(2)], nonnegativity, and a smoothness constraint. In other words, the present GWM directional resolution is roughly comparable to a directional wave buoy.

GWM (NOAA-WW3 hindcast) frequency-directional spectra for $1 \mathrm{yr}$ were used to estimate the first four loworder directional moments [(2)] in each frequency-time bin. Using MRM, MEM, and BDM (section 2), reconstructions of the GWM directional spectra were generated, and $R^{2}$ misfit of the reconstructed spectra to the original GWM spectra were computed. An example unimodal reconstruction by MRM and BDM is very good $\left(R^{2} \geq 0.9\right)$; however, performance declines as spectra complexity increases in the bimodal and trimodal cases (Fig. 7, left panels). Mean $R^{2}$ misfit values of MRM reconstructions for the entire year of GWM predictions show that high-energy events tend to have high $R^{2}>0.9$ across all frequencies (red in Fig. 8). Reconstructions of lower-energy directional spectra have lower skill, suggesting that spectra are more complex (green/blue in Fig. 8). The presented skills, with the exception of normalized skill in Fig. 4a, are more strongly influence by moderate to high energy events that are well represented by buoy directional moments. During these events GWM directional spectra provides little additional information, and therefore the combined (CMBADJ) and buoy-only methods have similar nearshore predictive skill.

The overall skill for buoy-only methods is comparable; however, we observed differences across metrics. MRM has lower $H_{\text {sig }}$ RMSE, MEM has lower directional bias, and BDM is a compromise of the two. No buoy-only method is the overall best performer, and likely the ability of each method to recreate the true directional spectra depends on frequency band, wave age, and region. GWM directional spectra predictions, used as a proxy for true spectra, yield insight into method differences and the importance of diffuse reflections and scattering (Fig. 7). Reconstructions of GWM directional spectra from the first four low-order moments using each buoy-only method (left panels) are contrasted with the addition of a small $(10 \%$ of the total energy) isotropic background energy added to the GWM spectra (right panels). Constant background energy (Snodgrass et al. 1966) crudely mimics diffuse shoreline reflections and scattering. Elgar et al. (1994) observed low levels of reflection from a natural beach, and Ardhuin and Roland (2012) found that constant reflection on the order of $10 \%$ improved GWM prediction along the coast (GWM predictions used here do not include reflection). The addition of background energy reduces MRM and BDM reconstruction skill but improves dramatically MEM reconstruction skill. MEM is designed to identify peak directions in the presence of background energy, its roots in astronomy, and image processing (Gull and Skilling 1984); however, peaks are too sharp when background energy is lacking. In contrast, MRM and BDM are both smoother than MEM, and background energy tends to overbroaden peaks. Additionally, in the bimodal case (Fig. 7d), the MRM and BDM peaks are slightly biased away from the overall mean. MEM better resolves incoming directional peaks in the presence of background energy, but BDM and MRM better resolve broader directional peaks with low background energy; no method is optimal. Buoy observations are fundamentally limited: these three methods are drawn from an infinite set (Ochoa and Delgado-González 1990), and each will perform only as well as their smoothness constraint represents the true underlying directional spectra. 
(a)

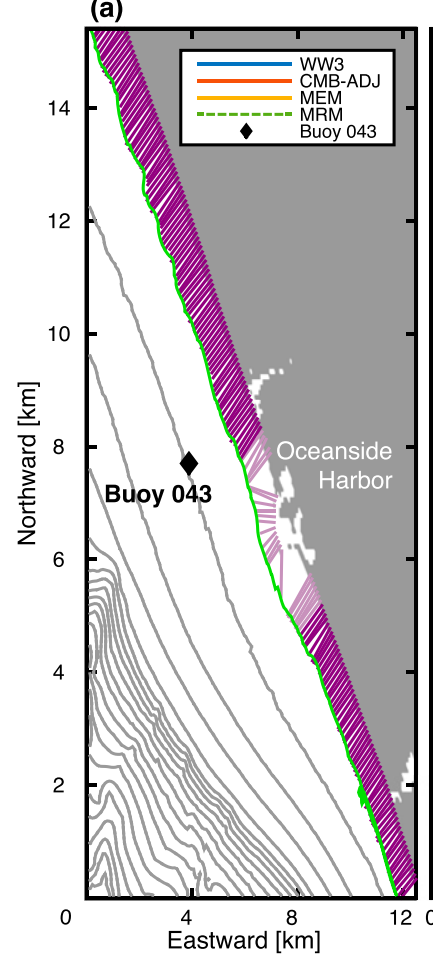

(b)

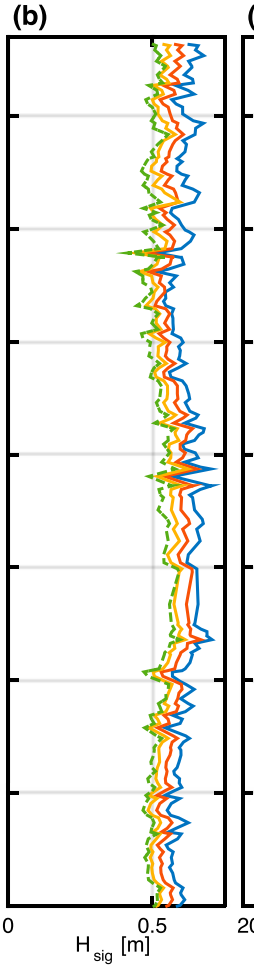

(c)

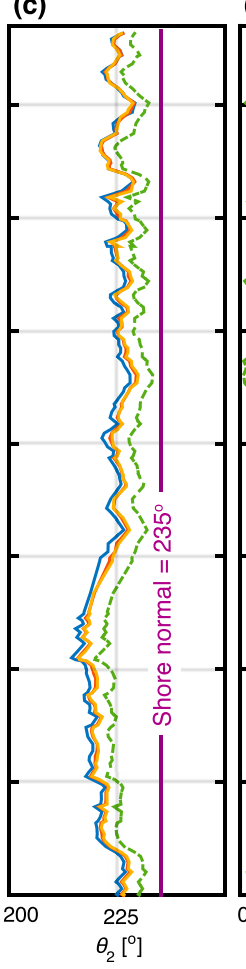

(d)

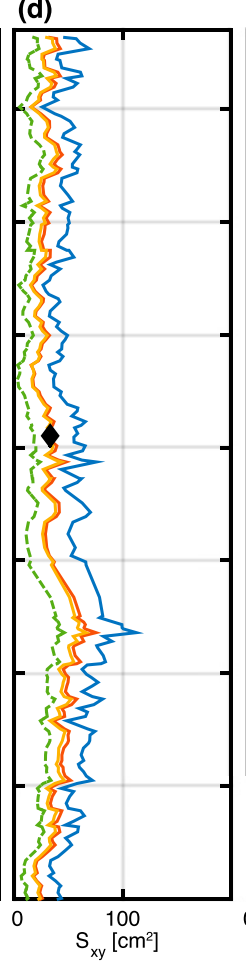

(e)

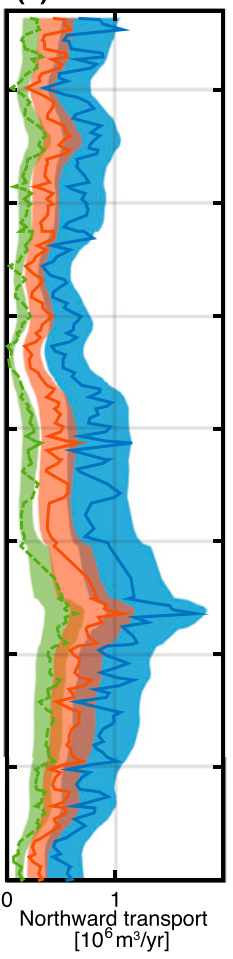

(f)

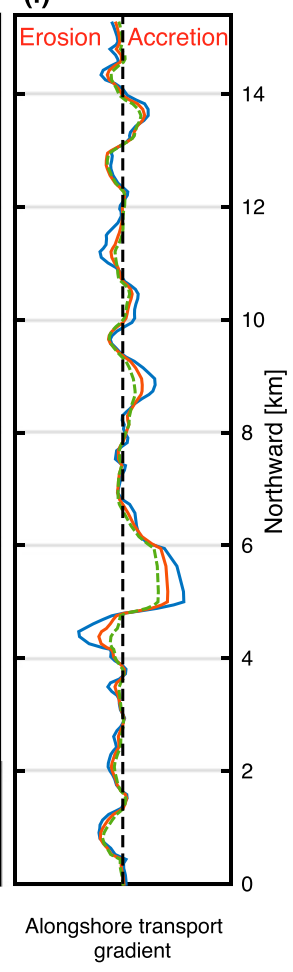

FIG. 6. (a) Plan view of Oceanside Harbor with buoy 043 (black diamond) and depth contours (gray curves; 20-m intervals). Local beach normal (magenta) are based on the nearest distance from the backshore point to 10-m depth contour (green curve). Predicted and observed time-averaged (b) $H_{\text {sig }}$ and (c) $\theta_{2}$ in 10-m depth vs alongshore distance for different boundary conditions (see legend). (d) Predicted mean $S_{x y}$ at 10-m depth vs alongshore distance is estimated using the alongshore mean shore normal (magenta) that excludes the harbor region, where shore normals are poorly defined [light magenta in (a)]. Buoy-measured mean $S_{x y}$ in 20-m depth is also shown (black diamond). (e) Predicted sediment transport using CERC formula, $K=0.6$, vs alongshore distance. Shaded colors show alongshoresmoothed (lowess 1.5 -km alongshore filter) transport estimates for the range of $K$ values $0.4<K<0.8$. (f) Gradient of lowess-filtered transport alongshore (e.g., the divergence of the drift) vs alongshore distance.

\section{b. Alongshore radiation stress and transport}

For most boundary condition methods, at most buoys, $\theta_{1}$ bias is $<10^{\circ}$, relatively small for most practical applications. However, at some buoy sites $S_{x y}$ estimates varied significantly (Fig. 5); at buoy sites 043 and 172, $S_{x y}$ estimates varied by more than a factor of 2 , substantial given that $S_{x y}$ is directly proportional to alongshore transport estimates based on the CERC formula. This variation in $S_{x y}$ from different boundary conditions exceeds the uncertainty in the CERC constant $K$ (Fig. 6f). Though boundary conditions utilizing offshore buoys yield better estimates of $S_{x y}$ than WW3, there is no overall best performer. The positive mean $S_{x y}$ at buoy 043, indicating northward transport, is contrary to expectations for the region (Inman and Frautschy 1965); however, this estimate is integrated only for swell energy $(0.04-0.09 \mathrm{~Hz})$. Mean $S_{x y}$ for the entire wave energy spectrum is typically negative (O'Reilly et al. 2016), indicating southward transport in the region.
Alongshore transport estimates from different boundary conditions differ primarily by a constant offset (Fig. 6e). Thus, the alongshore transport gradient-that is, divergence of the drift, indicating regions of accretion and erosion-is relatively consistent (Fig. 6f). Typically, the gradients of uncertain quantities are increasingly uncertain; however, in this case the bias between predictions in terms of contributing to $S_{x y}, H_{\text {sig }}$, and $\theta_{2}$ is constant alongshore (Figs. 6b,c). Therefore, accretion and erosion zones [(4)] may be determined confidently. We suspect that at these spatial scales $[O(1) \mathrm{km}]$, gradients in alongshore transport gradients are dominated by local bathymetry and high-order details of the offshore directional spectrum are insignificant. Note a single offshore normal is used to estimate $S_{x y}$, and smallscale variations in beach orientation are not included.

\section{c. Coastal reflection}

Weak coastal reflection $(<10 \%)$ has small effects on $H_{\text {sig }}$ and mean direction; even obliquely incident wave 
GWM
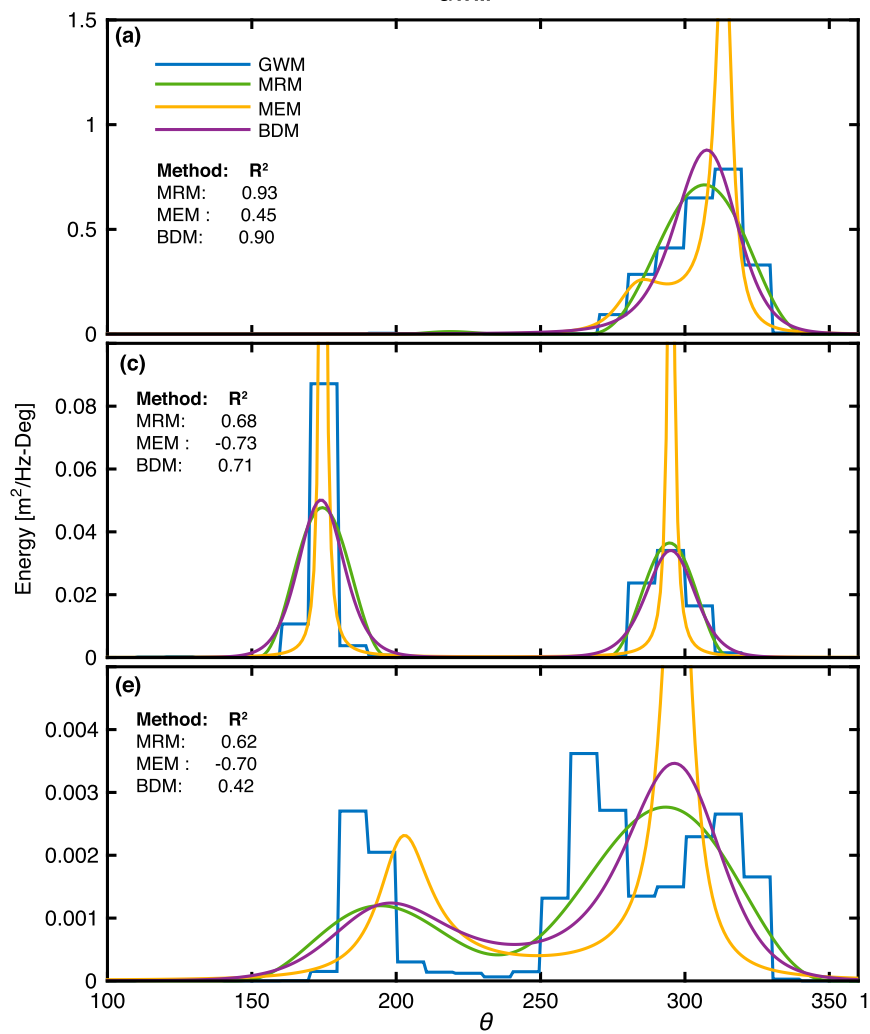

GWM + Isotropic Reflection

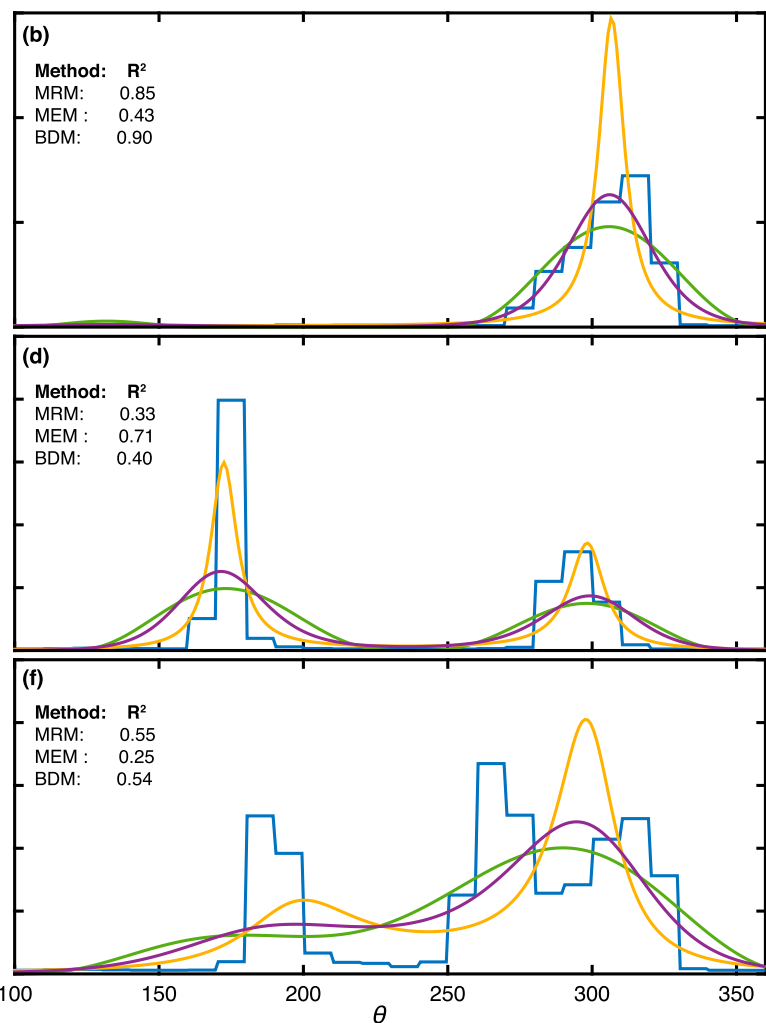

FIG. 7. Offshore wave energy vs direction for three cases: (a) and (b) unimodal, (c) and (d) bimodal, and (e) and (f) multimodal. (left) GWM (NOAA-WW3) directional spectra (blue) and recreations using various smoothness methods (see section 2) and loworder moments $\left[a_{1}, b_{1}, a_{2}, b_{2}\right.$ in (2)] integrated from the GWM spectrum. (right) GWM spectrum with an added isotropic signal equal to $10 \%$ of the total spectrum energy simulating diffuse wave reflection and corresponding smooth recreation. The $R^{2}$ (see tables) of the recreation and the original spectrum (GWM or GWM plus isotropic reflection) show that simple unimodal distributions are better recreated than multimodal shapes. The $R^{2}$ varies substantially in the presence of isotropic noise and between recreation methods.

conditions yield little change to $\theta_{1}$ and $\theta_{2}$ observations, less than $4^{\circ}$ and $2^{\circ}$, respectively. Alternatively, firstorder directional spread $\left(\sigma_{1}\right)$ observations are highly sensitive to coastal reflection. Including simple reflection parameterizations in GWMs significantly improves predicted $\sigma_{1}$ skill (Ardhuin and Roland 2012). While not included in our linear wave model, strong negative biases in $\sigma_{1}$ predictions across all model boundary conditions are consistent with weak coastal reflection (Table 2). Strong $\left(>10^{\circ}\right)$ tidal modulation of $\sigma_{1}$ is observed at local shallow buoy 172 offshore of Huntington Beach during a low-frequency event arriving from a high incident angle, $20^{\circ}-30^{\circ}$ southward of the local shore normal (Fig. 9b). At high tide, this convex beach has relatively high slope, and enhanced reflection, similar to Duck, North Carolina (Elgar et al. 1994). A buoy also senses the effect of directional narrowing due to increased refraction as the tide falls; however, these effects (estimated assuming plane-parallel contours;
Herbers et al. 1999) are small in 20-m depth for the local tidal amplitude $\left(<2^{\circ}\right.$; black line in Fig. $\left.9 \mathrm{~b}\right)$ and vanishingly small in more than roughly $100-\mathrm{m}$ depth. The observed modulation in $\sigma_{1}$ at buoy 172 corresponds roughly to a $3 \%-10 \%$ change in reflected energy, equivalent to a $2 \%-5 \%$ change in $H_{\text {sig }}$; similar levels of reflection are observed in Elgar et al. (1994). Owing to the high incident swell angle, tidal modulation of $\sigma_{2}$ is also observed, though at less than half the variance observed in $\sigma_{1}$. Coherence at tidal frequencies $\mathrm{M}_{2}$ and $\mathrm{K}_{2}$ between the local tidal elevation and observed $\sigma_{1}(f)$ (estimated independently at each observed frequency) is high in the swell band $(0.04-0.09 \mathrm{~Hz})$, peaking around $0.06 \mathrm{~Hz}$ and falling off at higher frequencies, where reflection is weaker (Fig. 9c). As expected for a nearby reflector, at local shallow buoys close to shore the phase lag for $\mathrm{K}_{1}$ and $\mathrm{M}_{2}$ frequencies is near zero $(< \pm 0.1 \mathrm{~h})$. At local deep buoys, the phase lags at $\mathrm{K}_{1}$ and $\mathrm{M}_{2}$ are scattered between $\pm 0.5 \mathrm{~h}$ (not shown). 


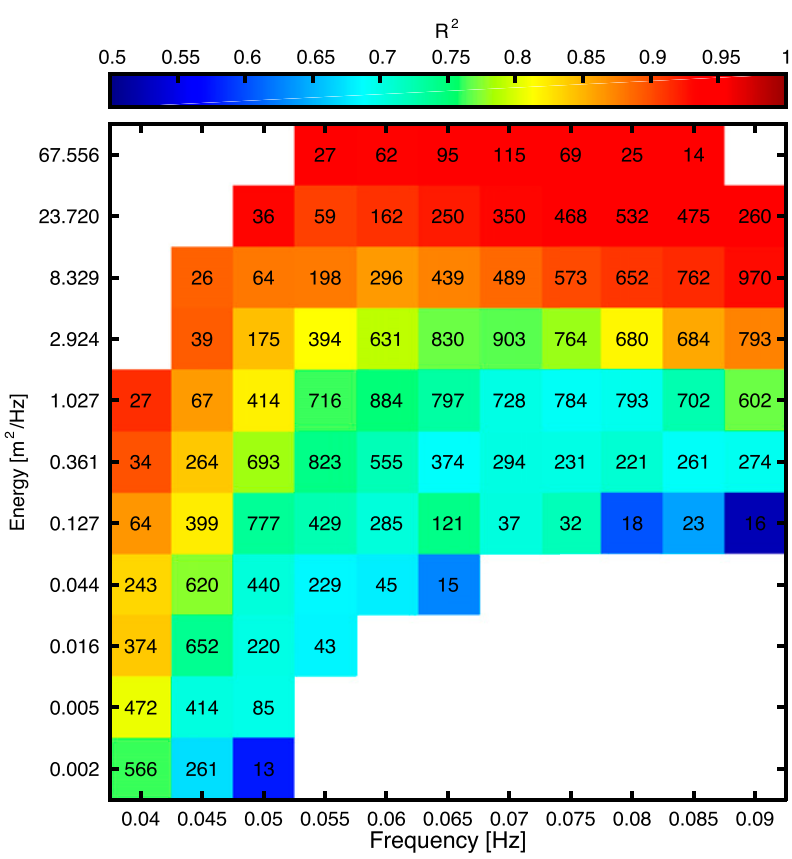

FIG. 8. Mean $R^{2}$ between GWM directional spectrum compared and recreation with second-order moments $\left[a_{1}, b_{1}, a_{2}, b_{2}\right.$ in (2)] and the MRM smoothness method (section 2) vs frequency ( $x$ axis) and offshore GWM energy ( $y$ axis). Black text is the number of 3-h GWM predictions in each frequency-energy bin for the 1 year (2006) of GWM predictions used. Note log scale for energy ( $y$-axis).

Steep coastline slopes reflect more incoming wave energy. The SC coastline is a mix of dissipative (fine to coarse sand), intermediate (gravel), and reflective (riprap, seawall, rocky cliffs) regions shown in Fig. 10 (NOAA 2006). The magnitude of $\sigma_{1}$ prediction bias (left half of colored square) at local buoy sites appears lower in regions with larger amounts of dissipative coastline, for example, San Diego (Fig. 10). Conversely prediction bias is larger where coastlines are steeper, for example, Santa Barbara and Los Angeles. This evidence is admittedly qualitative, and some observations suggest the opposite, e.g., buoy 092. Coherence of tidal elevation and $\sigma_{1}$ (colored right rectangle) is higher at shallow buoy sites and is significant at all buoys except 093 (Fig. 10). Coherence is lower further from the coastline because the travel times of reflected waves varies. At even larger distances (not shown), buoys are exposed to a large array of diffuse reflectors (coastline and islands), and tidal effects are blurred in direction and time (Snodgrass et al. 1966; Herbers et al. 1999).

\section{d. Santa Barbara Channel}

Our results confirm the previously observed large $H_{\text {sig }}$ prediction errors in the SBC (Rogers et al. 2007;
O'Reilly et al. 2016). The extreme sheltering by Point Conception and the Channel Islands (Fig. 1) amplifies the significance of high-order details of the offshore directional wave spectra. For example, during northwest wave events the SBC is often sheltered to the dominant swell direction by Point Conception, making critical the accurate description of the peak shoulder (e.g., Fig. S1 in the supplementary material). A higher-resolution boundary condition than provided by the WW3 predictions and buoy observations may be needed. Missing model physics likely partially explain these prediction errors; reflection from the steep cliff faces of the Channel Islands could help explain the $H_{\text {sig }}$ low bias. Also supporting reflection, the positive (northward) directional bias at buoy 107 suggests additional wave energy coming from a more southerly direction. However, further eastward in the SBC (buoys 111, 130,131) model directional bias suggests the opposite. It is not clear whether wave reflection contributes significantly to the large model errors in the SBC. Diffraction, surface current-induced refraction, and bathymetry inaccuracies may also contribute to prediction errors.

\section{Summary}

Different methods for parameterizing regional wave model offshore boundary conditions from buoy observations and global wave model GWM predictions (NOAA-WW3) are compared. Offshore wave energy was transformed to the nearshore with ray-tracingderived transfer coefficients and frequency-directiondependent time lags (section $3 \mathrm{~b}$ and appendix A). Offshore wave conditions were assumed homogenous in the along-crest direction for each discrete frequency and direction, a valid assumption for swell band $(0.04-0.09 \mathrm{~Hz})$ energy in SC (appendix A). Limiting model predictions to the swell band allowed for the use of point-based offshore information in the boundary condition, that is, buoy observations and frequency-directional GWM predictions. This methodology is valid for only swell energy in hindcast and nowcast modes.

Results show that for swell predictions, boundary conditions, including offshore buoys, performed better than those with GWM predictions alone. Buoyonly methods (MEM, BDM, MRM; refer to section 2) and the GWM-buoy combined method, CMB-ADJ, perform similarly, and they suggests that the directional "resolution" of low-order buoy moments is comparable to current GWM predictions. Supporting this hypothesis the complexity in directional spectra predictions for moderate to high energy events is well represented by low-order buoy directional moments 

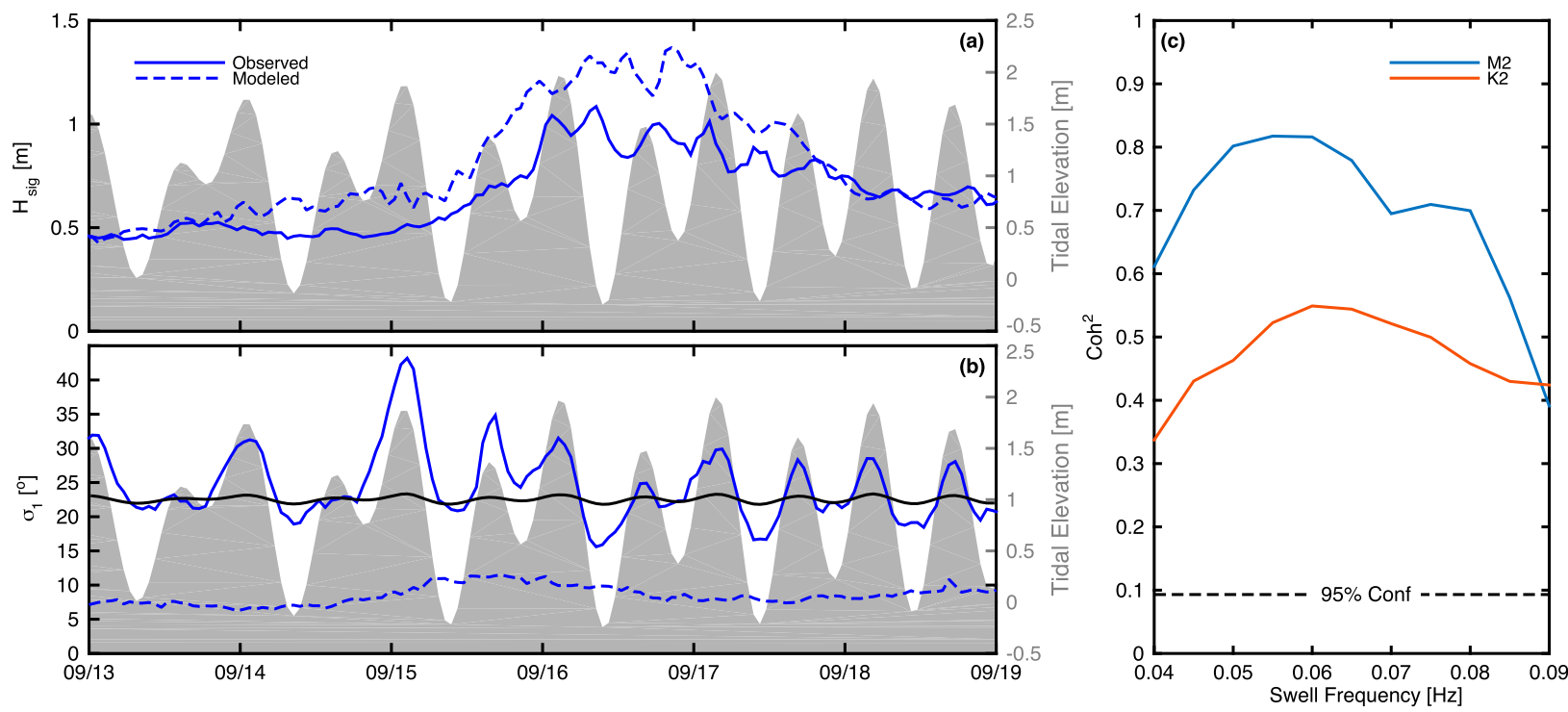

FIG. 9. The (a) $H_{\text {sig }}$ and (b) directional spread $\left(\sigma_{1}\right)$ vs time (UTC) for a low-frequency swell event $(f \sim 0.06 \mathrm{~Hz})$ at buoy 172 in 20 -m depth. Predicted tidal elevation in gray. The tidal modulation of $\sigma_{1}$ (solid blue), likely caused by coastal reflection, is not parameterized in modeled predictions (dashed blue, CMB-ADJ boundary condition). Modulation due to increased refraction at lower tidal levels (directional narrowing) is small, as shown for a constant $\sigma$ of $22.5^{\circ}$ (black). (c) Coherence between tidal elevation and $\sigma_{1}(f)$ at $\mathrm{M}_{2}$ and $\mathrm{K}_{2}$ tidal frequencies (see legend) for one complete year of buoy observations is significantly high for wave energy, where $0.04 \mathrm{~Hz} \leq f \leq 0.09 \mathrm{~Hz}$ ( $x$ axis). Black dashed line shows the 95\% coherence confidence level.

and smoothness assumptions (section 5a). Though GWM predictions may not currently add significant skill to buoy-only swell prediction, GWM predictions are necessary for higher-frequency energy that is affected by local winds and not along-crest homogenous offshore winds. It is not yet clear how to extend our simple combined approach to higher frequencies. However, as GWM predictions improve, the simple

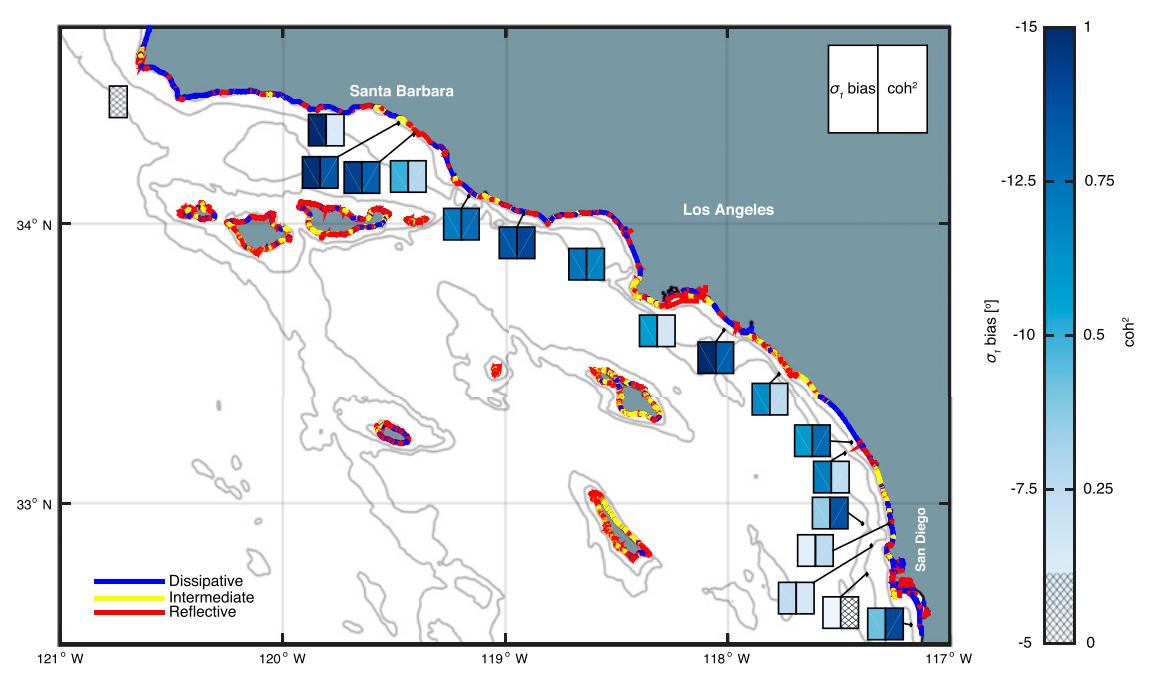

FIG. 10. Model (CMB-ADJ boundary condition) directional spread $\left(\sigma_{1}\right)$ prediction bias at local buoy sites shown in color (see color bar) in left half of square. Right half of square shows coherence at the $\mathrm{M}_{2}$ tidal frequency (1.9 cycles per day) of buoy-observed $\sigma_{1}(f=0.055 \mathrm{~Hz})$ and local tidal elevation (see color bar). Hatched colors indicate insignificant coherence levels at $95 \%$ confidence. The coastline is classified as dissipative (fine to coarse sand), intermediate (gravel), and reflective (riprap, rocky cliffs, seawall) types shown in color (see legend at bottom left). 
methodology in our CMB-ADJ method should yield higher skill for swell prediction, and it may also be a robust technique in varying regions and wave climates where buoy-only smoothness may fail.

For some applications-for example, recreational boating-our model errors are small. However, the variance in alongshore radiation stress predictions $\left(S_{x y}\right)$ was relatively high despite small $\left(<10^{\circ}\right)$ wave direction biases. Simple 1D (CERC) transport estimates varied twofold. However, despite large $S_{x y}$ uncertainties, the alongshore gradient of transport (e.g., the divergence of the drift) across all model predictions is surprisingly similar, suggesting that patterns of (theoretical) erosion and accretion may be insensitive to modest boundary condition errors and variations.

Acknowledgments. This study was funded by the California Department of Parks and Recreation, Division of Boating and Waterways Oceanography Program, the U.S. Army Corps of Engineers, and the California Sea Grant Traineeship Program. B. D. Cornuelle (Scripps Institution of Oceanography) provided valuable insight into inverse methods. Buoy data were obtained from the Coastal Data Information Program (CDIP; http://cdip.ucsd.edu/). C. B. Olfe (CDIP) helped with data and model management. R. Bucciarelli (CDIP) helped with coastline classification. We thank two anonymous reviewers for their many helpful suggestions.

\section{APPENDIX A}

\section{Model Transfer Coefficients}

For each nearshore location of interest-for example, buoy validation site-backward ray tracing relates the nearshore arrival angle $\theta$ to the offshore arrival $\theta^{(o)}$ (e.g., O'Reilly and Guza 1998, their Fig. 2). Rays are traced seaward following Snell's law as phase speed changes in varying bathymetry (Dobson 1967). Some rays terminate at offshore islands, indicating blocked arrival directions, while others are terminated when they reach deep water seaward of the SC Bight. The transfer coefficient $K^{2}$ is estimated using conservation of energy (depth-limited shoaling) and a simple relation from geometrical-optical theory such that,

$$
\frac{E(f, \theta)}{E^{(o)}(f, \theta)}=K^{2}(f, \theta)=\frac{c_{g}^{(o)}(f)}{c_{g}(f)}\left|\frac{\Delta \theta}{\Delta \theta^{(o)}}\right|
$$

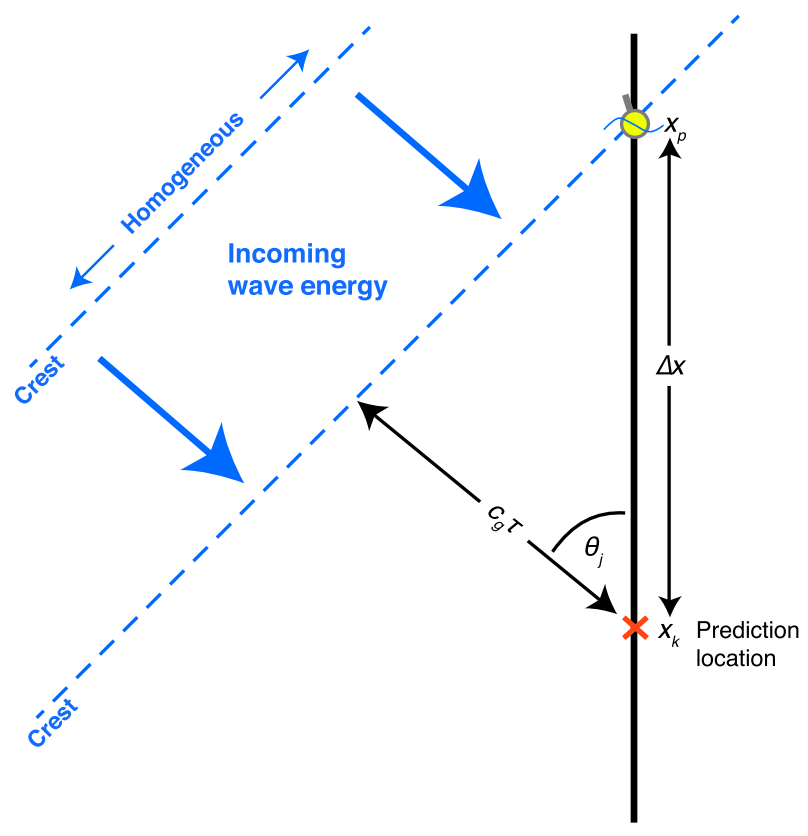

FIG. A1. Schematic of along-crest spatial homogeneity assumption and linear wave propagation at the group velocity $c_{g}$. Wave conditions (buoy observations or GWM predictions) at yellow symbol are used to predict waves at the red $x$. The time lag, $\tau(f, \theta, \Delta x)$, is used with complete frequency-directional spectra, $E(f, \theta, t)$, to make predictions at location $x_{k}$.

where $c_{g}$ is group velocity and $(o)$ indicates offshore properties (Dorrestein 1960). Mehaute and Wang (1982) show that (A1) is equivalent to Snell's law and the theoretical work of Longuet-Higgins (1957) for slowly varying bathymetry. Rays are traced at a very high directional resolution and carefully integrated to discretize $K^{2}$ at $1^{\circ}$ resolution (Dorrestein 1960). Unlike finite-difference propagation schemes, wave ray integration numerics are stable at any spatial/directional resolution and are only limited by the assumption of slowly varying bathymetry for linear shallow-water waves. Additionally, in this framework, $K^{2}$ is typically not a function of offshore position, assuming spatially homogenous and temporally stationary offshore conditions. Dorrestein (1960) provides a thorough discussion of this practical method for transforming wave energy nearshore.

\section{Offshore assumptions}

Low-frequency energy arriving from distant sources-for example, high southern and northern latitudes-is along-crest homogeneous on the scale of the SC domain $(300 \mathrm{~km})$, much less than the travel distance (Fig. A1). Additionally, at these spatial scales, low-frequency energy is assumed to 

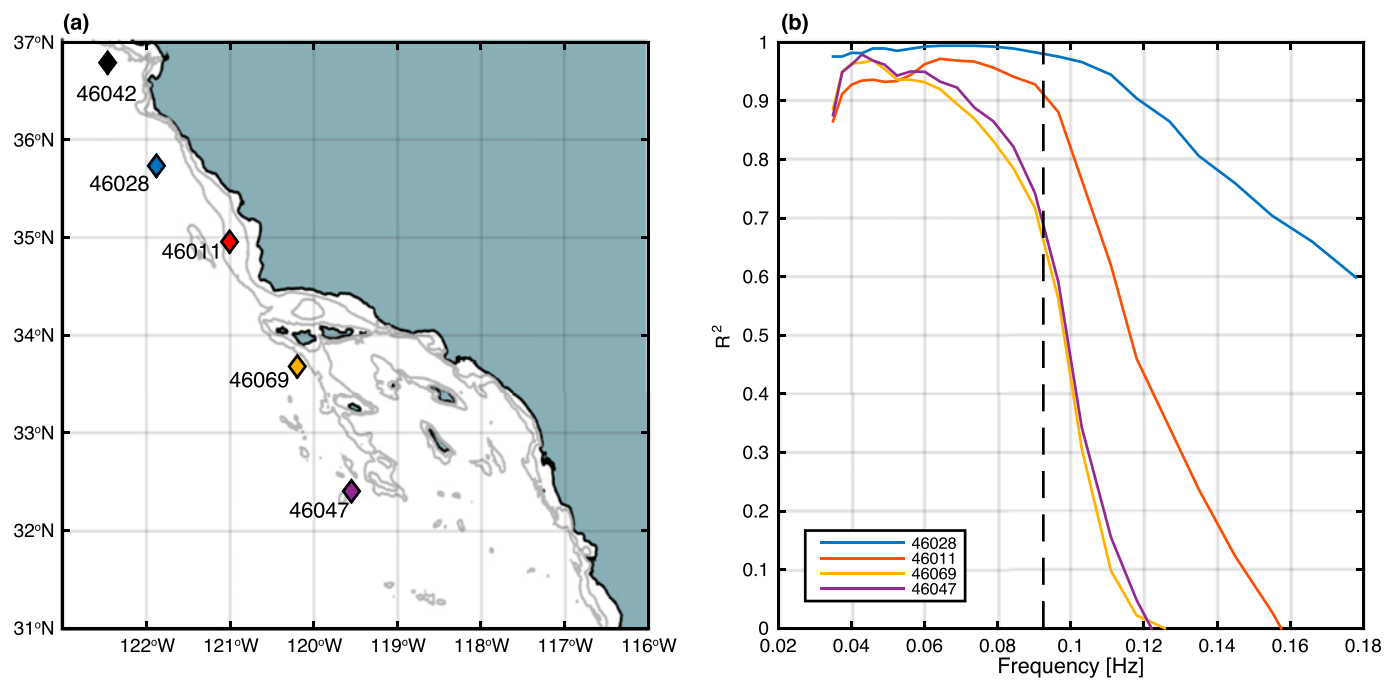

FIG. A2. (a) Map of Southern and central California showing locations of $E(f, \theta)$ GWM (NOAA-WW3) predictions for 2000-07 (NOAA buoy observations are not used here). GWM predictions at northerly buoy 46042 are used, with simple linear propagation and along-crest homogeneity assumptions [(A2)], to predict swell at the southerly sites. These model predictions are compared with the full frequency-directional GWM predictions at each site. (b) The $R^{2}$ skill vs frequency at southerly locations (see legend). The high prediction skill for frequencies $\leq 0.9 \mathrm{~Hz}$ (vertical dashed line) is similar for all approach directions (the average is shown) and supports the method of boundary condition specification used here.

propagate at the theoretical group velocity $c_{g}$ with negligible dissipation, generation, and nonlinear energy transfers. Under these assumptions, energy is transformed at each frequency and direction as

$E\left(f_{i}, \theta_{j}, x_{k}, t\right)=K^{2}(f, \theta) E\left[f_{i}, \theta_{j}, x_{p}, t+\tau\left(f_{i}, \theta_{j}, \Delta x\right)\right]$,

where $K^{2}$ captures the effect of bathymetry-induced refraction, shoaling, and island shadowing. The time lag $\tau$ for each location $\left(x_{k}\right)$, frequency, and direction is

$$
\tau\left(f_{i}, \theta_{j}, \Delta x\right)=\frac{\Delta x \cos \theta_{j}}{c_{g}\left(f_{i}\right)},
$$

and is illustrated in Fig. A1.

Assumptions (A2) and (A3) are tested offshore, with no bathymetry effects (i.e., $K^{2}=1$ ), using complete frequency-directional WW3 predictions, $F_{\text {Ww3 }}(f, \theta)$, that do not make these assumptions. Buoy observations, with inherent directional uncertainty (section 2), are less reliable for this test of assumptions. The $F_{\mathrm{Ww} 3}(f, \theta)$ predictions at NOAA buoy site 46042 (Fig. 6a) are used in (A2) and (A3) to predict wave conditions at buoy locations 46028 , 46011, 46069, and 46047. These linear predictions are compared with $F_{\mathrm{Ww} 3}(f, \theta)$ predictions at the buoy sites. The predictive skill of the assumptions in
(A2) and (A3) is shown in Fig. 6b using the $R^{2}$ metric. Skill declines sharply above $0.09 \mathrm{~Hz}$ for prediction sites farthest from buoy 46042 (Fig. 6) and was similar for varying wave directions (not shown). The high skill of (A2) and (A3) confirms the validity of both long-crest homogeneity and linear wave energy propagation assumptions for frequencies $<0.09 \mathrm{~Hz}$.

Here, these assumptions are used with transfer coefficients to predict waves nearshore; however, they can be additionally used to parameterize a typical regional wave model boundary with a singlepoint-based observation or GWM prediction. For example, locations along the thick black line in Fig. A2, representing a wave model boundary, could be well predicted using (A2) and (A3) for similar spatial scales $O(100) \mathrm{km}$ at similar frequencies $(0.04$ $0.09 \mathrm{~Hz})$.

\section{APPENDIX B}

\section{Metrics}

The metrics used in the paper are estimated from $N$ hour-length records. Given observations $o$ and predictions $p$, the following formulas are used: 


$$
\begin{aligned}
\text { RMSE } & =\sqrt{\frac{1}{N} \sum_{i=1}^{N}(p-o)^{2},} \\
\% \text { RMSE } & =\sqrt{\frac{1}{N} \sum_{i=1}^{N}\left(\frac{p-o}{o}\right)^{2},} \\
\text { bias } & =\frac{1}{N} \sum_{i=1}^{N}(p-o), \\
\% \text { bias } & =\frac{1}{N} \sum_{i=1}^{N} \frac{(p-o)}{o}, \\
R^{2} & =1-\frac{\sum_{i=1}^{N}(p-o)^{2}}{\sum_{i=1}^{N}(o-\bar{o})^{2}}, \\
r^{2} & =\frac{\left[\sum_{i=1}^{N}(o-\bar{o})(p-\bar{p})\right]^{2}}{\sum_{i=1}^{N}(o-\bar{o})^{2} \sum_{i=1}^{N}(p-\bar{p})^{2}},
\end{aligned}
$$

where the overbar indicates a mean. Detailed model results at each local buoy site are available in the supplementary material online.

\section{REFERENCES}

Adams, P. N., D. L. Inman, and N. E. Graham, 2008: Southern California deep-water wave climate: Characterization and application to coastal processes. J. Coastal Res., 244, 10221035, doi:10.2112/07-0831.1.

Ardhuin, F., and A. Roland, 2012: Coastal wave reflection, directional spread, and seismoacoustic noise sources. J. Geophys. Res., 117, C00J20, doi:10.1029/2011JC007832.

_ - and Coauthors, 2010: Semiempirical dissipation source functions for ocean waves. Part I: Definition, calibration, and validation. J. Phys. Oceanogr., 40, 1917-1941, doi:10.1175/ 2010JPO4324.1.

Backus, G. E., and J. F. Gilbert, 1967: Numerical applications of a formalism for geophysical inverse problems. Geophys. J. Int., 13, 247-276, doi:10.1111/j.1365-246X.1967.tb02159.x.

Boyd, S., and L. Vandenberghe, 2004: Convex Optimization. Cambridge University Press, $727 \mathrm{pp}$.

Camus, P., F. J. Mendez, and R. Medina, 2011: A hybrid efficient method to downscale wave climate to coastal areas. Coastal Eng., 58, 851-862, doi:10.1016/j.coastaleng.2011.05.007.

,,,--- A. Tomas, and C. Izaguirre, 2013: High resolution downscaled ocean waves (DOW) reanalysis in coastal areas. Coastal Eng., 72, 56-68, doi:10.1016/j.coastaleng.2012.09.002.

Chawla, A., and H. L. Tolman, 2008: Obstruction grids for spectral wave models. Ocean Modell., 22, 12-25, doi:10.1016/ j.ocemod.2008.01.003.

— D. Spindler, and H. L. Tolman, 2011: A thirty year wave hindcast using the latest NCEP Climate Forecast System Reanalysis winds. Proc. 12th Int. Workshop on Wave Hindcasting and Forecasting/Third Coastal Hazard Symp. Kona, HI, JCOMM, Environment Canada, and U.S. Army Corps of
Engineers, I1. [Available online at http://www.jcomm.info/ images/stories/2011/12thWaves/Papers/kona11_chawlaetal.pdf.]

— D. M. Spindler, and H. L. Tolman, 2013: Validation of a thirty year wave hindcast using the Climate Forecast System Reanalysis winds. Ocean Modell., 70, 189-206, doi:10.1016/ j.ocemod.2012.07.005.

Constable, S. C., R. L. Parker, and C. G. Constable, 1987: Occam's inversion: A practical algorithm for generating smooth models from electromagnetic sounding data. Geophysics, 52, 289-300, doi:10.1190/1.1442303.

Dobson, R. S., 1967: Some applications of the digital computer to hydraulic engineering problems. Tech. Rep., Dept. of Civil Eng., Standford University, 185 pp.

Dorrestein, R., 1960: Simplified method of determining refraction coefficients for sea waves. J. Geophys. Res., 65, 637-642, doi:10.1029/JZ065i002p00637.

Elgar, S., 1987: Bias of effective degrees of freedom of a spectrum. J. Waterw. Port Coastal Ocean Eng., 113, 77-82, doi:10.1061/ (ASCE)0733-950X(1987)113:1(77).

— T. H. C. Herbers, and R. T. Guza, 1994: Reflection of ocean surface gravity waves from a natural beach. J. Phys. Oceanogr., 24, 1503-1511, doi:10.1175/1520-0485(1994)024<1503: ROOSGW $>2.0 . \mathrm{CO} ; 2$.

García-Medina, G., H. T. Özkan-Haller, P. Ruggiero, and J. Oskamp, 2013: An inner-shelf wave forecasting system for the U.S. Pacific Northwest. Wea. Forecasting, 28, 681-703, doi:10.1175/WAF-D-12-00055.1.

Grant, M. C., and S. P. Boyd, 2013: CVX: Matlab software for disciplined convex programming. CVX Research, Inc. [Available online at http://cvxr.com/cvx.]

Gull, S. F., and J. Skilling, 1984: Maximum entropy method in image processing. IEE Proc. F, 131, 646-659, doi:10.1049/ ip-f-1:19840099.

Guza, R. T., E. B. Thornton, and N. Christensen Jr., 1986: Observations of steady longshore currents in the surf zone. J. Phys. Oceanogr., 16, 1959-1969, doi:10.1175/1520-0485(1986)016<1959: OOSLCI $>2.0 . \mathrm{CO} ; 2$.

Haas, K. A., and D. M. Hanes, 2004: Process based modeling of total longshore sediment transport. J. Coastal Res., 20, 853861, doi:10.2112/1551-5036(2004)20[853:PBMOTL]2.0.CO;2.

Hashimoto, N., and K. Konbune, 1988: Directional spectrum estimation from a Bayesian approach. Proceedings of 21st Conference on Coastal Engineering, B. L. Edge, Ed., Vol. 21, $62-76$.

Hayes, W. D., 1970: Conservation of action and modal wave action. Proc. Royal Soc. London, 320A, 187-208, doi:10.1098/ rspa.1970.0205.

Herbers, T. H. C., and R. T. Guza, 1990: Estimation of directional wave spectra from multicomponent observations. J. Phys. Oceanogr., 20, 1703-1724, doi:10.1175/1520-0485(1990)020<1703: EODWSF $>2.0 . \mathrm{CO} ; 2$.

_ - S. Elgar, and R. T. Guza, 1999: Directional spreading of waves in the nearshore. J. Geophys. Res., 104, 7683-7693, doi:10.1029/1998JC900092.

, P. F. Jessen, T. T. Janssen, D. B. Colbert, and J. H. MacMahan, 2012: Observing ocean surface waves with GPStracked buoys. J. Atmos. Oceanic Technol., 29, 944-959, doi:10.1175/JTECH-D-11-00128.1.

Inman, D. L., and J. D. Frautschy, 1965: Littoral processes and the development of shorelines. Coastal Engineering, ASCE, $511-536$

Kobune, K., and N. Hashimoto, 1985: Estimation of directional spectra from the maximum entropy principle. PARI Rep. 
024-03-04, Port and Harbour Research Institute, Ministry of Transport, 123-146.

Komar, P. D., and D. L. Inman, 1970: Longshore sand transport on beaches. J. Geophys. Res., 75, 5914-5927, doi:10.1029/ JC075i030p05914.

Kuik, A. J., G. P. van Vledder, and L. H. Holthuijsen, 1988: A method for the routine analysis of pitch-and-roll buoy wave data. J. Phys. Oceanogr., 18, 1020-1034, doi:10.1175/1520-0485(1988)018<1020: AMFTRA $>2.0 . \mathrm{CO} ; 2$

Lawson, L. M., and R. B. Long, 1983: Multimodal properties of the surface-wave field observed with pitch-roll buoys during GATE. J. Phys. Oceanogr., 13, 474-486, doi:10.1175/ 1520-0485(1983)013<0474:MPOTSW > 2.0.CO;2.

Long, R. B., 1980: The statistical evaluation of directional spectrum estimates derived from pitch/roll buoy data. J. Phys. Oceanogr., 10, 944-952, doi:10.1175/1520-0485(1980)010<0944: TSEODS $>2.0 . \mathrm{CO} ; 2$.

— , and K. Hasselmann, 1979: A variational technique for extracting directional spectra from multi-component wave data. J. Phys. Oceanogr., 9, 373-381, doi:10.1175/1520-0485(1979)009<0373: AVTFED $>2.0 . \mathrm{CO} ; 2$.

Longuet-Higgins, M. S., 1957: On the transformation of a continuous spectrum by refraction. Math. Proc. Cambridge Philos. Soc., 53, 226-229, doi:10.1017/S0305004100032163.

_ a physical discussion, with applications. Deep-Sea Res. Oceanogr. Abstr., 11, 529-562, doi:10.1016/0011-7471(64)90001-4.

—, D. E. Cartwright, and N. D. Smith, 1963: Observations of the directional spectrum of sea waves using the motions of a floating buoy. Ocean Wave Spectra: Proceedings of a Conference, Prentice-Hall, 111-136.

Lygre, A., and H. E. Krogstad, 1986: Maximum entropy estimation of the directional distribution in ocean wave spectra. J. Phys. Oceanogr., 16, 2052-2060, doi:10.1175/1520-0485(1986)016<2052: MEEOTD $>2.0 . C O ; 2$.

Mehaute, B. L., and J. D. Wang, 1982: Wave spectrum changes on a sloped beach. J. Waterw. Port Coastal Ocean Div., 108, 33-47.

Mínguez, R., A. Espejo, A. Tomás, F. J. Méndez, and I. J. Losada, 2011: Directional calibration of wave reanalysis databases using instrumental data. J. Atmos. Oceanic Technol., 28, 14661485, doi:10.1175/JTECH-D-11-00008.1.

NOAA, 2006: Sensitivity of coastal environments and wildlife to spilled oil. NOAA/Office of Response and Restoration.
Subset used: Shore types, accessed 2 November 2015. [Available online at ftp://ftp.dfg.ca.gov/R7_MR/HABITAT/.]

Ochoa, J., and O. E. Delgado-González, 1990: Pitfalls in the estimation of wind wave directional spectra by variationalprinciples. Appl. Ocean Res., 12, 180-187, doi:10.1016/ S0141-1187(05)80025-2.

Oltman-Shay, J., and R. T. Guza, 1984: A data-adaptive ocean wave directional-spectrum estimator for pitch and roll type measurements. J. Phys. Oceanogr., 14, 1800-1810, doi:10.1175/ 1520-0485(1984)014<1800:ADAOWD>2.0.CO;2.

O'Reilly, W. C., and R. T. Guza, 1993: A comparison of two spectral wave models in the Southern California Bight. Coastal Eng., 19, 263-282, doi:10.1016/0378-3839(93)90032-4. , and - 1998: Assimilating coastal wave observations in regional swell predictions. Part I: Inverse methods. J. Phys. Oceanogr., 28, 679-691, doi:10.1175/1520-0485(1998)028<0679: ACWOIR $>2.0 . C O ; 2$.

— , T. H. C. Herbers, R. J. Seymour, and R. T. Guza, 1996: A comparison of directional buoy and fixed platform measurements of Pacific swell. J. Atmos. Oceanic Technol., 13, 231-238, doi:10.1175/1520-0426(1996)013<0231:ACODBA > 2.0.CO;2.

_ - C. J. Olfe, R. J. Thomas, R. J. Seymour, and R. T. Guza, 2016: The California coastal wave monitoring and prediction system. Ocean Eng., 116, 118-132, doi:10.1016/ j.coastaleng.2016.06.005.

Orzech, M. D., J. Veeramony, and H. Ngodock, 2013: A variational assimilation system for nearshore wave modeling. J. Atmos. Oceanic Technol., 30, 953-970, doi:10.1175/JTECH-D-12-00097.1.

Panteleev, G., M. Yaremchuk, and E. W. Rogers, 2015: Adjointfree variational data assimilation into a regional wave model. J. Atmos. Oceanic Technol., 32, 1386-1399, doi:10.1175/ JTECH-D-14-00174.1.

Rogers, E. W., J. M. Kaihatu, L. Hsu, R. E. Jensen, J. D. Dykes, and K. T. Holland, 2007: Forecasting and hindcasting waves with the SWAN model in the Southern California Bight. Coastal Eng., 54, 1-15, doi:10.1016/j.coastaleng.2006.06.011.

Snodgrass, F. E., G. W. Groves, K. F. Hasselmann, G. R. Miller, W. H. Munk, and W. H. Powers, 1966: Propagation of ocean swell across the Pacific. Philos. Trans. Roy. Soc. London, 259, 431-497, doi:10.1098/rsta.1966.0022.

Tolman, H. L., 2009: User manual and system documentation of WAVEWATCH III version 3.14. MMAB Tech. Rep. 276, NOAA, 220 pp. 